\title{
Genome-wide analysis of in vivo CcpA binding with and without its key co-factor HPr in the major human pathogen group A
}

\author{
Streptococcus
}

Sruti DebRoy ${ }^{1}$, Victor Aliaga Tobar ${ }^{3,4}$, Gabriel Galvez ${ }^{4}$, Srishtee Arora ${ }^{5}$, Xiaowen Liang ${ }^{5 \#, ~ N i c o l a ~ H o r s t m a n n ~}{ }^{1}$, Vinicius Maracaja-Coutinho ${ }^{3,9}$, Mauricio Latorre ${ }^{4,6,7,8}$, Magnus Hook ${ }^{5}$, Anthony R. Flores ${ }^{10,11}$ and Samuel A. Shelburne ${ }^{1,2,11^{*}}$

${ }^{1}$ Department of Infectious Diseases Infection Control and Employee Health, ${ }^{2}$ Department of Genomic Medicine, University of Texas MD Anderson Cancer Center, Houston TX, USA, ${ }^{3}$ Facultad de Ciencias Químicas y Farmacéuticas, Advanced Center for Chronic DiseasesACCDiS, Universidad de Chile,Santos Dumont 964, Independencia, Santiago,Chile, 4 Laboratorio de Bioingeniería, Instituto de Ciencias de la Ingeniería, Universidad de O’Higgins, Libertador Bernardo O'Higgins 611, Rancagua, O'Higgins, Chile, ${ }^{5}$ Center for Infectious and Inflammatory Diseases, Institute of Biosciences and Technology, Texas A\&M University Health

Science Center, Houston, TX, USA; ${ }^{6}$ Laboratorio de Bioinformática y Expresión Génica, INTA, Universidad de Chile, El Libano 5524, Macul, Santiago, Chile, ${ }^{7}$ Mathomics, Center for Mathematical Modeling, Universidad de Chile, Beauchef 851, 7th Floor, Santiago, Santiago, Chile, ${ }^{8}$ Center for Genome Regulation (Fondap 15090007), Universidad de Chile, Blanco 
Encalada 2085, Santiago, Santiago, Chile, ${ }^{9}$ Centro de Modelamiento Molecular, Biofísica y Bioinformática (CM2B2), Facultad de Ciencias Químicas y Farmacéuticas, Universidad de Chile, Santiago, Chile, ${ }^{10}$ Division of Infectious Diseases, Department of Pediatrics, ${ }^{11}$ Center for Antimicrobial Resistance and Microbial Genomics, University of Texas Health Science Center McGovern Medical School, Houston, TX, USA.

*Corresponding author: Samuel A. Shelburne, MD, PhD, Department of Infectious Diseases, MD Anderson Cancer Center, Unit 1460, 1515 Holcombe Blvd, Houston, TX, 77030, 713-7923629 (o), 713-792-5381 (fax), sshelburne@mdanderson.org

\#Current Address: Xiaowen Liang, Department of Experimental Therapeutics, University of Texas MD Anderson Cancer Center, Houston TX, USA

Running title: Global CcpA binding in Streptococcus pyogenes

Keywords: Streptococcus pyogenes, ChIPseq, HPr-independent CcpA regulation

Data sharing and data availability: The data that support the findings of this study are available from the corresponding author upon reasonable request. 


\section{Summary}

Catabolite control protein A (CсрA) is a master regulator of carbon source utilization and contributes to the virulence of numerous medically important Gram-positive bacteria. Most functional assessments of CcpA, including interaction with its key co-factor HPr, have been performed in nonpathogenic bacteria. In this study we aimed to identify the in vivo DNA binding profile of CcpA and assess the extent to which HPr is required for CcpA-mediated regulation and DNA binding in the major human pathogen group A Streptococcus (GAS). Using a combination RNAseq/ChIPseq approach, we found that CcpA affects transcript levels of 514 of 1667 GAS genes (31\%) whereas direct DNA binding was identified for 105 GAS genes. Three of the directly regulated genes encode the key GAS virulence factors Streptolysin S, PrtS (IL-8 degrading proteinase), and SpeB(cysteine protease). Mutating CcpA Val301 to Ala (strain 2221-CcpA-V301A) abolished interaction between CcpA and HPr and impacted the transcript levels of 205 genes (40\%) in the total CcpA regulon. By ChIPseq analysis, CcpAV301A bound to DNA from $74 \%$ of genes bound by wild-type CcpA, but generally with lower affinity. These data delineate the direct CcpA regulon and clarify the HPr-dependent and independent activities of CcpA in a key pathogenic bacterium. 


\section{$\underline{\text { Introduction }}$}

Carbon catabolite repression (CCR) is a global process by which bacteria prioritize the use of favorable energy sources (Deutscher et al., 2006, Fujita, 2009). The core mechanism of CCR is an alteration in levels of proteins involved in metabolite transport and utilization which in turn is primarily achieved at the transcriptional level (Gorke \& Stulke, 2008). The LacI-GalR family transcriptional regulator catabolite control protein (CcpA) is a key mediator of CCR in many Gram-positive bacteria (Henkin et al., 1991, Titgemeyer \& Hillen, 2002, Warner \& Lolkema, 2003). CcpA inactivation impacts $\sim 15-20 \%$ of the transcriptome of a broad array of Gram-positive bacteria with the majority of impacted genes encoding proteins involved in carbohydrate and nitrogen utilization (Antunes et al., 2012, DebRoy et al., 2016, Seidl et al., 2009, Zeng et al., 2013). Importantly, CcpA also affects the transcript levels of genes encoding known and putative virulence factors in human Gram-positive pathogens ranging from streptococci to Clostridia (Iyer et al., 2005, Mertins et al., 2007, Seidl et al., 2008a, Varga et al., 2004). Consequently, CcpA inactivation in diverse organisms result in altered virulence-related phenotypes such as extracellular capsule production, biofilm formation, lysis of red blood cells, and inter-species signaling (Giammarinaro \& Paton, 2002, Johnson et al., 2009, Kinkel \& McIver, 2008, Seidl et al., 2008b, Watson et al., 2013).

Although CсрA is clearly critical to the virulence of numerous key Gram-positive pathogens, understanding of CcpA physiologic function is mainly derived from studies in non-pathogenic bacteria (Warner \& Lolkema, 2003). Based primarily on investigations in Bacillus species, CcpA is currently thought to impact gene expression by binding cis-acting DNA known as catabolite response elements (cre) which are composed of the pseudo-palindromic motif WTGNAANCGNWNNCWW (where W= A or $\mathrm{T}$ and $\mathrm{N}$ = any base) (Miwa et al., 2000, Schumacher et al., 2011, Stulke \& Hillen, 2000). CcpA affinity for cre sites is significantly increased by the co-effector molecule, histidine-containing protein (HPr) phosphorylated at Ser46 (HPrSer46 P) (Aung-Hilbrich et al., 2002, Deutscher et al., 2005, Shelburne et al., 2008). HPr is phosphorylated and dephosphorylated at Ser46 by HPr kinase/phosphorylase (HPrK/P), a bifunctional ATP-dependent enzyme whose activity is responsive to 
intracellular energy status (Poncet et al., 2004). Thus, the interaction of CcpA with HPr facilitates alteration in gene expression in response to metabolic changes (Deutscher et al., 2006). All major, invasive Gram-positive pathogens contain highly conserved orthologues of CcpA, HPr, and HPrK/P with amino acid similarities ranging from 70\% for CcpA to 85\% for HPr. However, Bacillus species also contain Crh (catabolite repression HPr), which is an HPr-like protein important for CcpA-mediated gene regulation that is not present in typical Gram-positive pathogens such as staphylococci and streptococci (Deutscher et al., 2006, Galinier et al., 1997, Schumacher et al., 2006).

Although the pseudo-palindromic cre sequence seems to be the predominate site of CcpA-DNA interaction in Bacillus species, there have been suggestions that CcpA may bind other DNA sites. Using in silico approaches, cre sites have been predicted in only a small percentage of genes whose transcript levels are signific antly altered by CcpA inactivation (Carvalho et al., 2011, DebRoy et al., 2016). Additionally, a recent study in Clostridium acetobutylicum identified a CcpA binding motif (TGTAA/TTTACA) which is quite distinct from the previous cre motif (Yang et al., 2017). Moreover, in addition to the "classic" cre motif, a genome-wide CcpA binding study identified a cre2 motif in Streptococcus suis (TTTTYHWDHHWWTTTY, where Y is C or T, H is A or C or T, and D is A or G or T) that was primarily important for CcpA function in the stationary phase (Willenborg et al., 2014). Although CcpA transcriptome analyses have been performed in a wide variety of bacteria, genome-wide characterization of CcpA-DNA binding is not currently available for a major, invasive Gram-positive pathogen other than Streptococcus suis (Antunes et al., 2012, Buescher et al., 2012, Willenborg et al., 2014).

In addition to a sub-optimal understanding of how CcpA interacts with DNA in invasive Grampositive bacteria, analysis of the role of HPr and HPrK/P in pathogenic bacteria has been quite limited (Mertins et al., 2007, Shelburne et al., 2008). In part, this is because in major Gram-positive pathogens such as group A Streptococcus (GAS), Streptococcus pneumoniae, and Staphylococcus aureus, HPr or even HPrSer46 P appears to be essential, in contrast to what is observed in Bacillus species (Fleming et al., 2015). In Staphylococus xylosus elimination of HPrSer46 P completely abolished CCR suggesting 
that CcpA absolutely requires HPrSer46 P to impact gene transcription (Jankovic \& Bruckner, 2002). In contrast, CcpA regulation at cre2 sites in S. suis was postulated to be independent of HPr (Willenborg et al., 2014). As pathogens establish and propagate human infections, they are likely to encounter vastly different metabolic conditions, which in turn would be expected to alter HPrSer46 P levels. Thus establishing whether CcpA can function independently of HPrSer46 P and which genes are regulated by CcpA in the absence of HPrSer46 P is highly pertinent to fully understand the role that nutritional acquisition plays in modulation of pathogenesis and infection outcomes. Herein, we sought to determine the global DNA binding characteristics of CcpA in GAS and analyze the effect of blocking the interaction between CcpA and HPrSer46 P in order to broaden insight into the physiology underlying the critical contribution of CcpA to Gram-positive pathogenesis. 


\section{$\underline{\text { Results }}$}

A CсpA mutant that does not bind $\mathrm{HPr}$

We chose to conduct our study in the emm1 GAS strain MGAS2221 because emm1 strains are leading causes of GAS infections, MGAS2221 is representative of the current emm1 strains causing human disease worldwide, and MGAS2221 has a fully sequenced genome and lacks mutations in known regulators that have been shown to impact the CcpA transcriptome (Shelburne et al., 2010, Sumby et al., 2005). Both HPr and HPrK/P are essential in GAS (Le Breton et al., 2015). Thus, to probe into the possible existence of a CcpA regulon independent of HPrSer46 P, we first attempted to generate an HPrS46A mutant. However, we were unable to generate a viable GAS HPr-S46A mutant despite repeated efforts suggesting that, as in the case of Streptococcus pneumoniae (Fleming et al., 2015), a S46A mutation in GAS HPr is lethal, possibly due HPrSer46 P dependent cellular functions that are essential. As an alternate approach we sought to generate a GAS mutant which retains the ability for HPr to be phosphorylated, but does not permit interactions between HPrSer46 P and CcpA. In silico alanine scanning mutagenesis of the CcpA-HPrSer46 P interface, modeled on the crystal structure of the Bacillus megaterium CcpA-HPrSer46 P-DNA complex, identified four amino acid residues of CcpA that are critical to CcpA-HPrSer46 P interaction (Homeyer et al., 2007, Schumacher et al., 2004). We chose to alter one of these residues, valine 301, to alanine given that the V301 residue is conserved in all CcpA family proteins (Schumacher et al., 2004) and gene regulation studies have demonstrated that a CcpAV301 mutant is compromised in glucose-mediated gene repression (Sprehe et al., 2007).

Table 1. Strains and plasmids used in this study.

\section{Strain or plasmid}

\section{Description}

\section{Reference}

\section{Strains}

MGAS2221

$2221 \Delta c c p A$

2221-СcрA-V301A
Invasive clinical isolate, reference serotype M1

MGAS2221 $\triangle c c p A:: s p c$

MGAS2221 with V301A change in CcpA
(Sumby et al., 2006)

(Shelburne et al., 2010)

This study 
BL21-pET-His2-CcpA E. coli producing recombinant wild type GAS CcpA (Shelburne et al., 2008)

BL21-pET-His2-V301A E. coli producing recombinant GAS CcpA with This study

BL21-pET-His2-HPr $\quad$ E. coli producing recombinant GAS HPr

(Shelburne et al., 2010)

BL21-pET21a-HPrKP E. coli producing recombinant GAS HPrK/P $\quad$ (Shelburne et al., 2010)

\section{Plasmids}

pET-His2-CcpA pET-His2 plasmid with GAS cсpA gene

pET-His2-V301A pET-His2 plasmid with GAS ccpA gene encoding V301A change

(Shelburne et al., 2008)

This study

pET-His2-HPr pET-His2 plasmid with GAS HPr gene

(Shelburne et al., 2010)

pET21a-HPrK/P pET21a plasmid with GAS HPrK/P gene

(Shelburne et al., 2010)

pBBL740 ссрAV301A

pBBL740 plasmid with GAS cсpA gene encoding V301A change

This study

We first sought to test the hypothesis that the CcpAV301A mutation would impair the interaction of recombinant CcpA and HPr using surface plasmon resonance (SPR). To this end, we expressed and purified recombinant CcpA and CcpAV301A along with HPr. We used recombinant HPrK/P to phosphorylate HPr at Ser46 as previously described (Shelburne et al., 2010). As expected, wild type CcpA had about 30 fold higher affinity (i.e. bound more tightly) for HPrSer $46 \sim$ P relative to $\mathrm{HPr}$ with $K_{\mathrm{D}}$ value of $5.8 \pm 0.5$ vs. $188 \pm 18 \mu \mathrm{M}$ (Figure 1). Consistent with our hypothesis that V301 is critical for CcpA-HPrSer46 P interaction in GAS, recombinant CcpAV301A bound to HPrSer46 P with a $K_{\mathrm{D}}$ value of $153 \pm 17 \mu \mathrm{M}$ which closely approximated that observed for wild type CcpA/HPr interaction $\left(K_{\mathrm{D}}=188\right.$ $\pm 18 \mu \mathrm{M})$ (Figure 1). The interaction between CcpAV301A and HPr is much weaker and the affinity could not be determined under the conditions used. The equilibrium dissociation constant values $\left(K_{\mathrm{D}}\right)$ are mean \pm standard deviation obtained from two experiments. 
A

\section{HPrSer46 P $\Rightarrow$ CcpA}

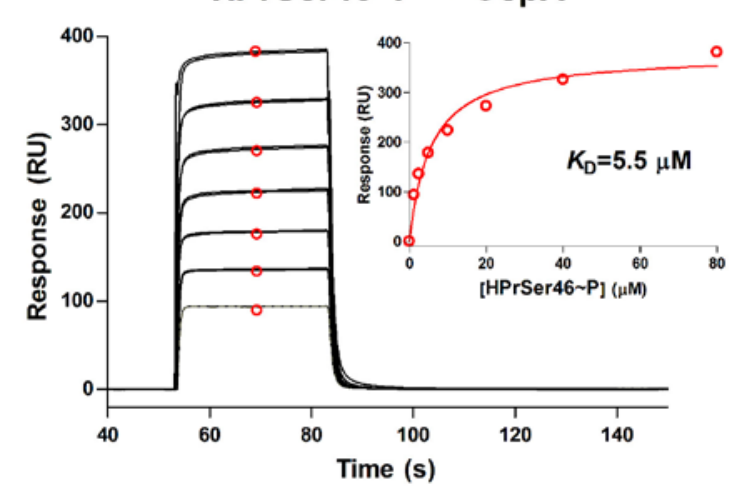

C

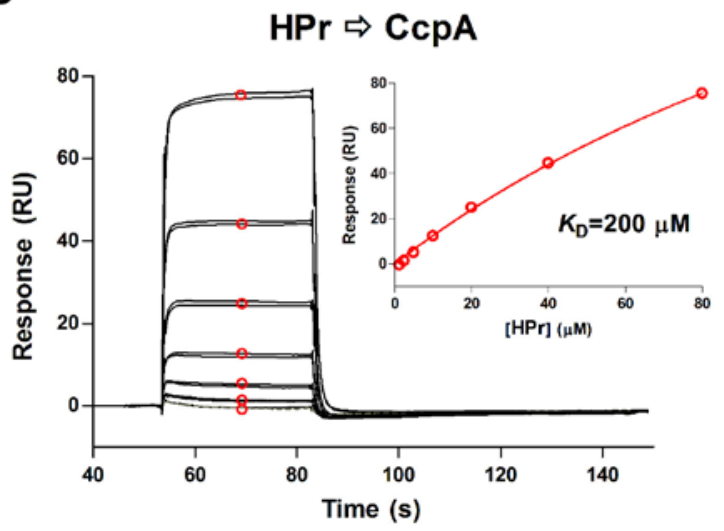

B

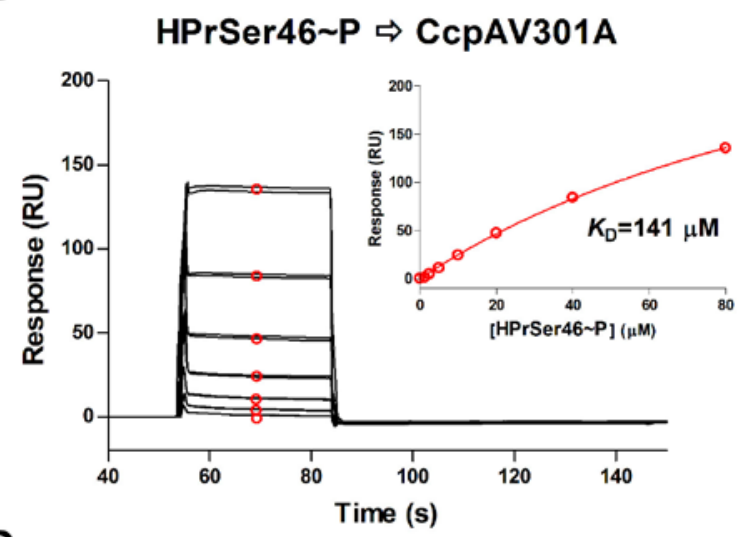

D

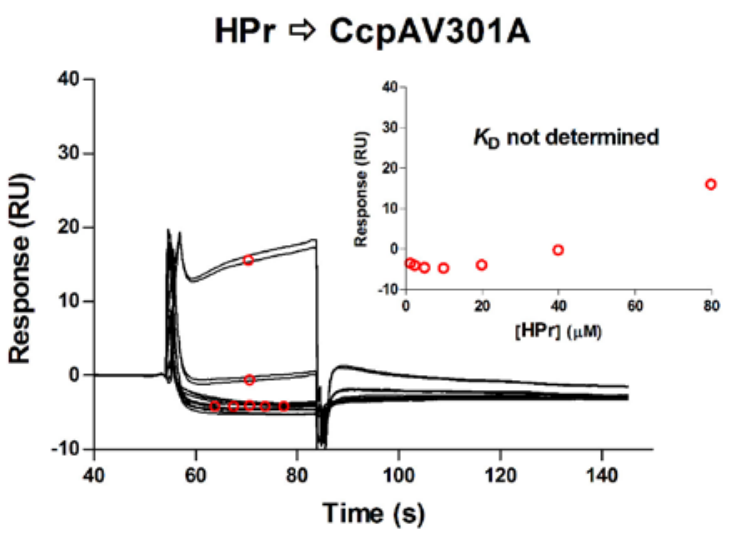

Figure 1. In vitro analysis of CcpA-HPr interaction. Representative SPR analysis $(n=2)$ of the binding between CcpA and HPr recombinant proteins. HPrSer46 P and HPr (2-fold serial dilutions from 1.25 to $80 \mu \mathrm{M}$ ) was injected in duplic ate to (A \& C) CcpA surface (3700 RU) and (B \& D) CcpAV301A surface (4200 RU). The SPR response curves of bound protein are shown in black with lower curve corresponding to lower concentration of protein injected. The average responses at steady state (shown in red circles) were plotted as a function of the HPr concentration and the isotherm was fit to a one-site binding (hyperbola) model (fitted curve shown in red) to determine equilibrium dissociation constant $K_{\mathrm{D}}$ (inset).

Next, we used site directed mutagenesis to create the isoallellic mutant strain 2221-CcpA-V301A in the same parental background, the serotype emm1 strain MGAS2221, as our previously created 2221 $\Delta c c p A$ isolate (Table 1). Given that deleting ссрA has previously been shown to affect $\mathrm{HPr} / \mathrm{HPr} \sim \mathrm{P}$ 
ratios in other bacteria (Leboeuf et al., 2000, Ludwig et al., 2002), we used Phos-tag gels to analyze HPr and $\mathrm{HPr} \sim \mathrm{P}$ levels at the mid-exponential phase of growth in a nutrient rich media. Under these conditions, the majority of HPr in strains MGAS2221 and 2221-CcpA-V301A was unphosphorylated with no significant difference of $\mathrm{HPr} / \mathrm{HPr} \sim \mathrm{P}$ ratios identified between the two strains (Figure 2A, B). Conversely, HPr P levels were increased in strain $2221 \Delta c c p A$ relative to the other two strains. A similar increase in $\mathrm{HPr} \sim \mathrm{P}$ levels has been observed in ccpA mutants of $B$. subtilis and $E$. faecalis, and has been attributed to increased HPr kinase activity (Leboeuf et al., 2000, Ludwig et al., 2002). These data show that the V301A alteration in CcpA did not have significant effects on cellular HPr P levels (Figure 2A, B). Additionally, the cellular levels of CcpA were similar between MGAS2221 and 2221-CcpA-V301A indicating that the amino acid variation did not impact CcpA autoregulation (note similar CcpA band densities for MGAS2221 and 2221-CcpA-V301A in Figure 2C, third panel).

A

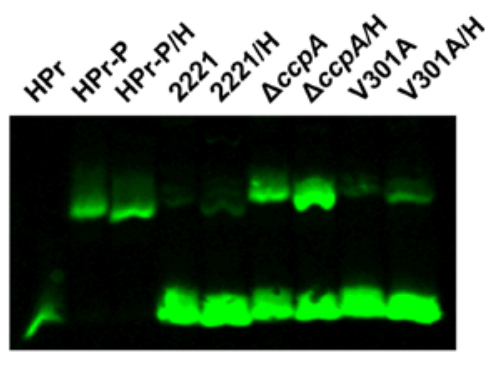

B

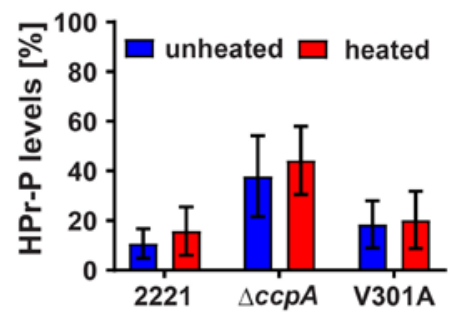

C

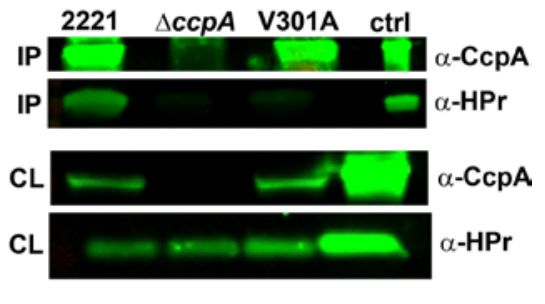

Figure 2. Characterization of the CcpAV301A mutant protein. (A) Representative Phostag Western blot ( $\mathrm{n}=2$ ) and (B) graphical representation of the levels of phosphorylated HPr in lysates of wild type (2221), ccpA deletion mutant ( $\triangle c c p A)$ and the CcpAV301A mutant (V301A) grown to mid-exponential phase. Lanes marked with "H” indicate heated samples. Purified recombinant HPr and HPr P was used as controls. Error bars indicate standard deviation. (C) Co-immunoprecipitation of HPr from lysates of indicated strains using anti-CcpA antibody. "IP" and "CL” indicates immunoprecipitated material and cell lysate respectively. The antibody for each sub-panel is indicated on the right. Purified recombinant CcpA and HPr proteins were used as controls (ctrl). 
To further test our hypothesis that CcpAV301A was not interacting with HPr in vivo, we performed immunoprecipitation reactions using anti-CcpA antibody which detects both the wild type and CcpAV301A mutant protein (Figure 2C, first and third panels). Strains MGAS2221, 2221 $\Delta c c p A$, and 2221-CcpA-V301A were crosslinked and harvested at mid-exponential phase. CcpA-containing complexes were immunoprecipitated using a polyclonal anti-CcpA antibody and then analyzed for the presence of HPr by western blotting using anti-HPr antibody (Figure 2C). By quantitative analysis, 10 times more HPr was immunoprecipated by CcpA antibody from strain MGAS2221 compared to strain 2221-CcpA-V301A. No HPr was immunoprecipated by CcpA antibody in strain $2221 \Delta c c p A$. Taken together, we conclude that the V301A amino acid change essentially abolishes the ability of CcpA to interact with HPr both in vitro and in vivo.

\section{Inability of CcpA to interact with HPr impacts the CcpA transcriptome}

Given that CcpA-HPrSer46 P interaction is thought to be critical for CcpA activity (Deutscher et al., 2005, Deutscher et al., 2006, Deutscher, 2008, Gorke \& Stulke, 2008, Stulke \& Hillen, 2000), we next sought to test the hypothesis that the CcpAV301A change would result in a transcriptome similar to that observed for a complete CcpA knockout. To this end, we performed RNAseq analyses, in quadruplicate, for strains MGAS2221, 2221 $\Delta c c p A$, and 2221-CcpA-V301A grown to mid-exponential phase in THY. We did not observe any significant changes in ccpA transcript levels between MGAS2221 and 2221CcpA-V301A, confirming that the V301A change does not affect transcription of ccpA itself (Supplementary Figure S1). Principal component analysis showed that the data were reproducible and, contrary to our hypothesis, that the transcriptomes of the three strains were quite distinct (Figure 3A). We assigned transcript levels as being signific antly different when there was a mean difference of at least 1.5 fold and a P $<0.05$ after accounting for multiple comparisons. We first sought to assess how the transcriptome of MGAS2221differed from 2221 $\Delta c c p A$. Deletion of $c c p A$ resulted in signific antly different transcript levels of 514 genes representing 31\% of the 1667 genes in the MGAS2221 genome 
with sufficient transcript levels for analysis (Supplementary Table 1). More genes (361) had increased transcript levels in strain $2221 \Delta c c p A$ (i.e. were CcpA repressed) compared to genes (153) whose transcript levels were lower in $2221 \Delta c c p A$ (i.e. were CcpA activated), which is in accordance with previous observations (Carvalho et al., 2011, DebRoy et al., 2016, Kietzman \& Caparon, 2011, Shelburne et al., 2008). The transcript levels of twelve virulence factor encoding genes were impacted by CcpA inactivation including speB, speA2, sic, spd, ska, grab, the sag operon, the nga-slo operon, and prtS (Table 2). Consistent with the central role of CcpA in carbon source acquisition and utilization, the transcript levels of genes encoding 13/14 of the phosphotransferase systems (PTS) present in MGAS2221 were significantly altered by CcpA inactivation as were genes encoding four ATP binding cassette (ABC) carbohydrate transporters (Table 3). Cluster of orthologous group (COG) analysis revealed that genes whose transcript levels were significantly impacted by CcpA inactivation were more likely to be in groups C (energy production and conversion) and G (carbohydrate transport and metabolism) relative to unaffected genes (Figure 3B).

Next, we analyzed transcript levels in strain 2221-CcpA-V301 for genes whose transcript levels were significantly different between strains MGAS2221 and 2221 $\Delta c c p A$. If the CcpA-HPr interaction is critical to regulation of a particular CcpA-impacted gene then we would expect that transcript levels for said gene to be significantly different between strains MGAS221 and 2221-CcpA-V301A. However, of the 514 genes whose transcript levels were signific antly affected by CcpA inactivation, only 205 (40\%) also had significantly different transcript levels in strain 2221-CcpA-V301A relative to strain MGAS2221 (Figure 3C). We considered these to be HPr-dependent genes because eliminating the interaction between CcpA and Hpr impacted their transcript levels (Supplementary Table 1). The remaining 309 (60\%) genes whose transcript levels were impacted by CcpA inactivation did not have significantly different transcript levels between strains MGAS2221 and 2221-CcpA-V301A and were therefore denoted as HPr-independent (Figure 3C) (Supplementary Table 1). A significantly higher percentage of the HPr-dependent genes were CcpA repressed (157/205, 77\%) compared to HPr-independent genes (204/309, 66\%, $\mathrm{P}=0.01$ by Fisher's exact test). For the twelve virulence factor encoding genes/operons 
impacted by CcpA inactivation, only four were HPr-dependent (nga-slo, sag operon, grab, and endoS). Conversely, of the seventeen CcpA-regulated genes/operons encoding carbohydrate transport systems, fourteen were HPr-dependent (Table 2, 3). Similarly, COG analysis showed that, the HPr-dependent genes were more likely to be in category G (carbohydrate transport and metabolism) and less likely to be in category L (replication and repair), M (cell wall/membrane/envelop biogenesis), and S (function unknown) relative to HPr-independent genes (Figure 3B).

A

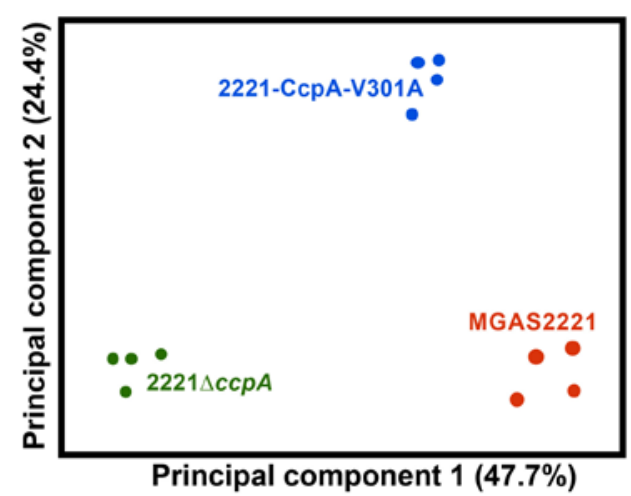

B

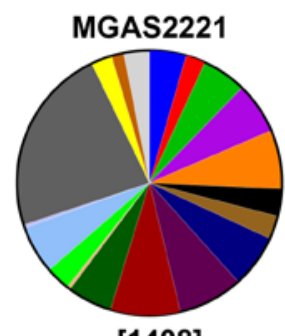

[1408]

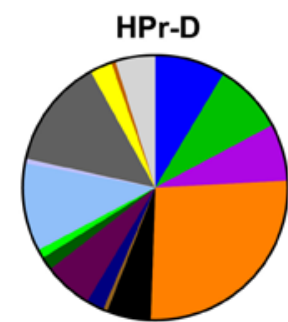

[186] $\triangle c c p A / M G A S 2221$

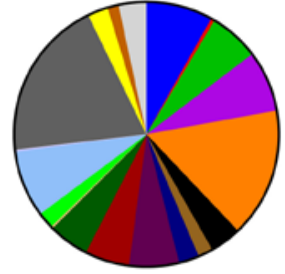

[443]

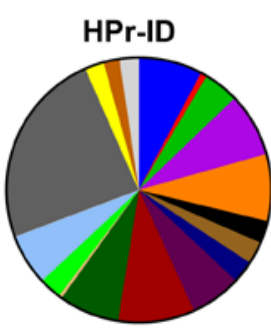

[257]
C

$2221 \triangle \mathrm{ccpA} / \mathrm{MGAS} 2221$

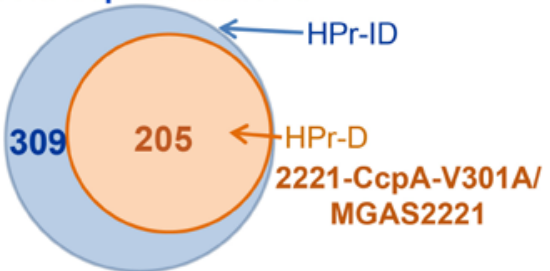

D

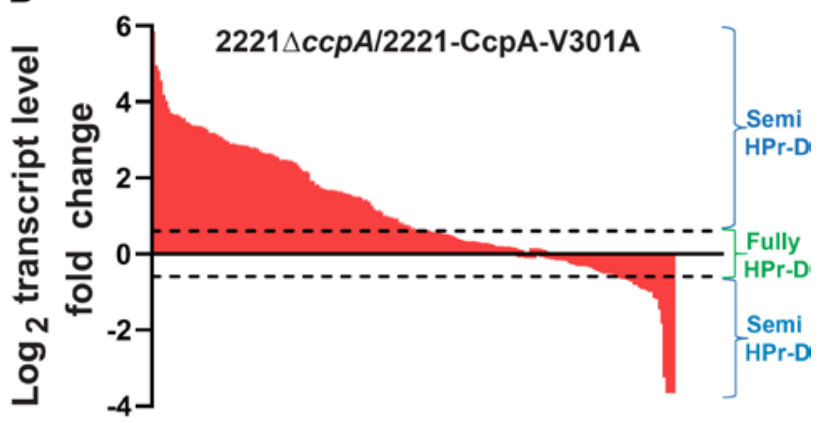

E

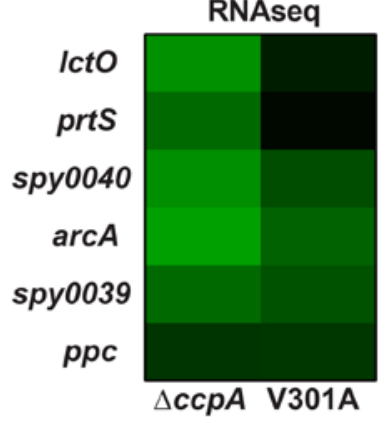

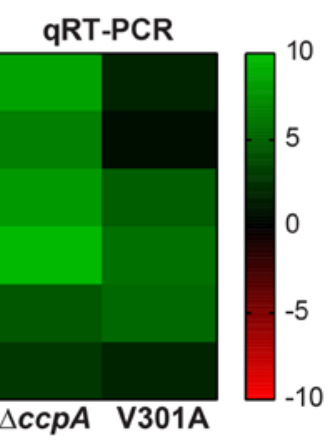

Figure 3. Impact of CcpAV301A mutation on the GAS transcriptome. (A) Principal component analysis showing that the transcriptomes of MGAS2221, 2221 $\triangle с c p A$ and 2221-CcpA-V301A are distinct. 
Each strain has four biological replicates. (B) COG-based distribution of all genes in MGAS2221, genes impacted by cсpA deletion ( $\triangle c c p A / M G A S 2221)$ and the HPr-dependent (HPr-D) and HPr-independent (HPr-ID) genes. [C] Energy production and conversion; [D] Cell cycle control, cell division, chromosome partitioning; [E] Amino acid transport and metabolism; [F] Nucleotide transport and metabolism; [G] Carbohydrate transport and metabolism; [H] Coenzyme transport and metabolism; [I] Lipid transport and metabolism; [J] Translation, ribosomal structure and biogenesis; [K] Transcription; [L] Replication, recombination and repair; [M] Cell wall/membrane/envelope biogenesis; [N] Cell motility; [O] Posttranslational modification, protein turnover, chaperones; [P] Inorganic ion transport and metabolism; [Q] Secondary metabolites biosynthesis, transport, and catabolism; [S] Function unknown; [T] Signal transduction mechanisms; [U] Intracellular trafficking, secretion, and vesicular transport and [V].Defense mechanisms. (C) Venn diagram showing the subsets of $c c p A$-affected genes that are HPrindependent (HPr-ID) and HPr-dependent (HPr-D). The strain comparisons used to generate these subsets are indicated in their respective colors. (D) Waterfall plot showing the gradation in the magnitude of the transcriptional impact of the CcpAV301A mutation on the HPr-dependent genes. The fold change for HPr-dependent genes between strains 2221 $\Delta c c p A$ and 2221-CcpA-V301A, as observed in the RNAseq, is plotted. The genes within the dotted line are fully HPr-dependent, while those outside are HPr semi-dependent. (E) Gene transcript levels of selected CcpA-impacted genes that exhibit varying transcriptional effects of the CcpAV301A mutation by RNAseq analysis were validated by targeted Taqman qRT-PCR analysis. Transcript levels in strains 2221 $\Delta c c p A$ and 2221-CcpA-V301A are shown relative to wild type MGAS2221. For Taqman qRT-PCR, data are mean \pm standard deviation of two biological replicates, with two technical replicates, done on two separate days $(n=8)$.

We next sought to determine whether the loss of CcpA-HPr interaction was equivalent to complete CcpA inactivation in terms of the absolute effect on gene expression by using fold-change (FC) comparison of the HPr-dependent genes. If the CcpA-HPr complex is essential for CcpA-regulation of a 
particular gene, then there should be no significant difference in transcript levels between strains 2221 $\Delta c c p A$ and 2221-CcpA-V301A. In fact, similar transcript levels in these two strains were observed for only 78 (38\%) of the $205 \mathrm{HPr}$-dependent genes, which we subsequently considered as fully HPrdependent (i.e. the effect of eliminating CcpA-HPr interaction was equivalent to the effect of total CcpA inactivation) (Supplementary Table 1). For the remaining 127/205 (62\%) genes, transcript levels were significantly different between strains $2221 \Delta c c p A$ and 2221-CcpA-V301A. Given that CcpA is primarily a repressor, for these 127 genes the loss of interaction with HPr resulted in transcript levels that were generally lower than complete CcpA inactivation, but the magnitude of effect varied for individual genes (Figure 3D). We designated these genes as semi-HPr-dependent (Supplementary Table 1). All four HPrdependent virulence factor encoding genes/operons (nga-slo operon, streptolyin S operon, grab, and endoS) were semi-HPr-dependent (Table 2). Of the 14 HPr-dependent carbohydrate utilization encoding operons, only two (lactose and fructose transporters) were fully HPr-dependent (Table 3). We chose CcpA-regulated genes that exhibit varying degrees of transcriptional impact upon disrupting the CcpAHPr interaction and verified the changes in transcript levels using targeted qRT-PCR (Figure 3E). Taken together, our data show that CcpA influences a signific ant proportion of the GAS genome even without HPr interaction and that the quantitative impact of HPr on CcpA-mediated regulation is distinct for different genes.

Table 2. Influence of CcpA-HPr interaction on virulence gene regulation and DNA binding.

\begin{tabular}{|c|c|c|c|c|c|c|}
\hline $\begin{array}{c}\text { M5005 } \\
\text { spy\# }\end{array}$ & Gene & $\begin{array}{c}\text { CcpA } \\
\text { repressed } \\
\text { vs } \\
\text { activated }\end{array}$ & $\begin{array}{c}\text { HPr } \\
\text { dependent vs. } \\
\text { independent }\end{array}$ & $\begin{array}{c}\text { If HPr } \\
\text { dependent : } \\
\text { semi or fully }\end{array}$ & $\begin{array}{c}\text { Bound } \\
\text { by } \\
\text { CcpA }\end{array}$ & $\begin{array}{c}\text { Bound by } \\
\text { CcpAV301A }\end{array}$ \\
\hline \hline $0139-0141$ & nga-slo & Repressed & Dependent (nga) & Semi & No & No \\
0341 & prtS & Repressed & Independent & Yes & Yes \\
0351 & spyA & Repressed & Independent & No & No
\end{tabular}




$\begin{array}{|ccccccc|}\text { 0562-70 } & \begin{array}{c}\text { Streptolysin S } \\ \text { operon }\end{array} & \text { Repressed } & \text { Dependent } & \text { Semi } & \text { Yes } & \text { Yes } \\ 0996 & \text { speA2 } & \text { Repressed } & \text { Independent } & & \text { No } & \text { No } \\ 1106 & \text { grab } & \text { Activated } & \text { Dependent } & \text { Semi } & \text { No } & \text { No } \\ 1540 & \text { endoS } & \text { Activated } & \text { Dependent } & \text { Semi } & \text { No } & \text { No } \\ 1684 & \text { ska } & \text { Repressed } & \text { Independent } & & \text { No } & \text { No } \\ 1711 & \text { Imb } & \text { Repressed } & \text { Independent } & & \text { No } & \text { No } \\ 1718 & \text { sic } & \text { Repressed } & \text { Independent } & & \text { No } & \text { No } \\ 1735 & \text { speB } & \text { Activated } & \text { Independent } & & \text { Yes } & \text { Yes } \\ 1738 & \text { spd } & \text { Repressed } & \text { Independent } & & \text { No } & \text { No }\end{array}$

Table 3. Influence of CcpA-HPr interaction on regulation and DNA binding of genes encoding carbohydrate transport systems of MGAS2221.

\begin{tabular}{|c|c|c|c|c|c|c|}
\hline $\begin{array}{l}\text { M5005 } \\
\text { spy\# }\end{array}$ & $\begin{array}{l}\text { Putative transported } \\
\text { carbohydrate }\end{array}$ & $\begin{array}{c}\text { Transporter } \\
\text { type }^{a}\end{array}$ & $\begin{array}{c}\text { CcpA } \\
\text { repressed } \\
\text { vs } \\
\text { activated }\end{array}$ & $\begin{array}{c}\text { HPr } \\
\text { dependent } \\
\text { vs. } \\
\text { independent }\end{array}$ & $\begin{array}{c}\text { Bound } \\
\text { by } \\
\text { CcpA }\end{array}$ & $\begin{array}{c}\text { Bound by } \\
\text { CcpAV301A }\end{array}$ \\
\hline 0212-0219 & Sialic acid & $\mathrm{ABC}$ & Repressed & Independent & Yes & No \\
\hline 0475 & B-glucoside & PTS & Repressed & $\begin{array}{c}\text { Semi- } \\
\text { dependent }\end{array}$ & No & No \\
\hline 0521 & $\mathrm{~N}$-acetylgluc osamine & PTS & Repressed & Independent & No & No \\
\hline 0662 & Fructose & PTS & Repressed & $\begin{array}{c}\text { Fully - } \\
\text { dependent }\end{array}$ & No & No \\
\hline 0780-0783 & Mannose/fructose & PTS & Repressed & $\begin{array}{c}\text { Semi- } \\
\text { dependent }\end{array}$ & No & No \\
\hline $1058-1060$ & Maltose & $\mathrm{ABC}$ & Repressed & $\begin{array}{c}\text { Semi- } \\
\text { dependent }\end{array}$ & Yes & Yes \\
\hline 1067-1062 & Maltodextrin & $\mathrm{ABC}$ & Repressed & $\begin{array}{c}\text { Semi- } \\
\text { dependent }\end{array}$ & Yes & Yes \\
\hline 1083-1079 & Cellobiose & PTS & Repressed & $\begin{array}{c}\text { Semi- } \\
\text { dependent }\end{array}$ & Yes & Yes \\
\hline $1310-1308$ & Unknown sugar & $\mathrm{ABC}$ & Repressed & Semi- & No & No \\
\hline
\end{tabular}




\begin{tabular}{|c|c|c|c|c|c|c|}
\hline \multirow[b]{2}{*}{ 1399-1401 } & \multirow[b]{2}{*}{ Galactose } & \multicolumn{5}{|c|}{ dependent } \\
\hline & & PTS & Repressed & $\begin{array}{c}\text { Semi- } \\
\text { dependent }\end{array}$ & No & No \\
\hline 1479-1481 & Mannose & PTS & Repressed & $\begin{array}{c}\text { Semi- } \\
\text { dependent }\end{array}$ & Yes & Yes \\
\hline 1542 & Sucrose & PTS & Activated & $\begin{array}{c}\text { Semi- } \\
\text { dependent }\end{array}$ & No & No \\
\hline 1634-1633 & Lactose & PTS & Activated & $\begin{array}{c}\text { Fully - } \\
\text { dependent }\end{array}$ & No & No \\
\hline 1664-1662 & Mannitol & PTS & Repressed & $\begin{array}{c}\text { Semi- } \\
\text { dependent }\end{array}$ & No & No \\
\hline 1692 & Maltose & PTS & Repressed & Independent & Yes & Yes \\
\hline 1746-1744 & Cellobiose & PTS & Repressed & $\begin{array}{c}\text { Semi- } \\
\text { dependent }\end{array}$ & Yes & No \\
\hline 1784 & Trehalose & PTS & Repressed & $\begin{array}{c}\text { Semi- } \\
\text { Dependent }\end{array}$ & No & No \\
\hline
\end{tabular}

aPTS - phosphotransferase system; ABC - ATP binding cassette

In vivo interaction of CcpA and CcpAV301A with GAS chromatin

There are limited genome wide assessments of CcpA binding to DNA in pathogenic bacteria and none for a strain in which CcpA-HPr interaction has been abrogated (Willenborg et al., 2014). Therefore, we performed ChIP-Seq analysis for strains MGAS2221 and 2221-CcpA-V301A grown to midexponential phase in THY media to analyze CcpA chromatin occupancy profiles and assess how the reduced ability of CcpA to interact with HPr affects CcpA-DNA binding. The 2221 $\triangle c c p A$ strain was used as a control to ensure the specificity of our findings.

We identified 76 CcpA binding sites in strain MGAS2221, including in the promoter region of ссрA itself which is consistent with a previous in vitro analysis of CcpA binding (Almengor et al., 2007) (Table 4). The genome-wide distribution of these sites is shown in Figure 4A. The lack of significant enrichment at any of these sites in strain $2221 \triangle c c p A$ indic ates that these are true in vivo binding sites for the CcpA protein. The CcpA binding sites were mostly (67\%) in predicted promoter regions (i.e. within 
500 bps of a translational start site) (Fujita, 2009), which is in accord with other CcpA ChIP-Seq analyses (Antunes et al., 2012, Buescher et al., 2012, Willenborg et al., 2014). Of the 76 enriched regions, 46 sites (61\%) were associated with genes whose transcript levels were significantly altered in strain $2221 \Delta c c p A$ relative to strain MGAS221. These 46 sites were associated with 45 operons containing 107 genes. The discrepancy between numbers of binding sites and operons affected is due to the occasional identification of multiple distinct binding sites for the same gene. Thus, only 107 (21\%) of the 514 genes whose transcript levels were significantly impacted by CcpA inactivation in strain MGA2221were identified as being directly regulated by CcpA (Figure 4B). The vast majority of sites enriched in the ChIPseq analysis (88\%) correlated with genes which had increased transcript levels in strain $2221 \Delta c c p A$ relative to MGAS2221 (i.e. were CcpA-repressed). By COG analysis, genes directly regulated genes by CcpA were significantly more likely to be in category C (energy production and conversion) and G (carbohydrate transport and metabolism) (Figure 4C).

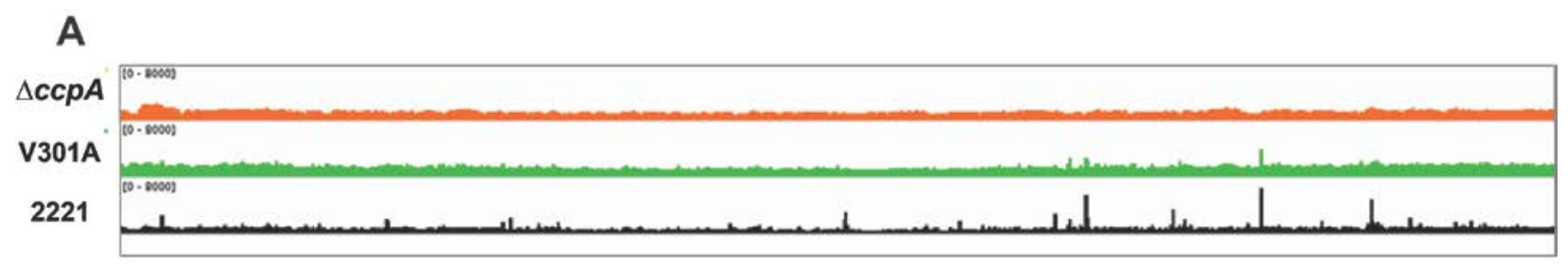

B

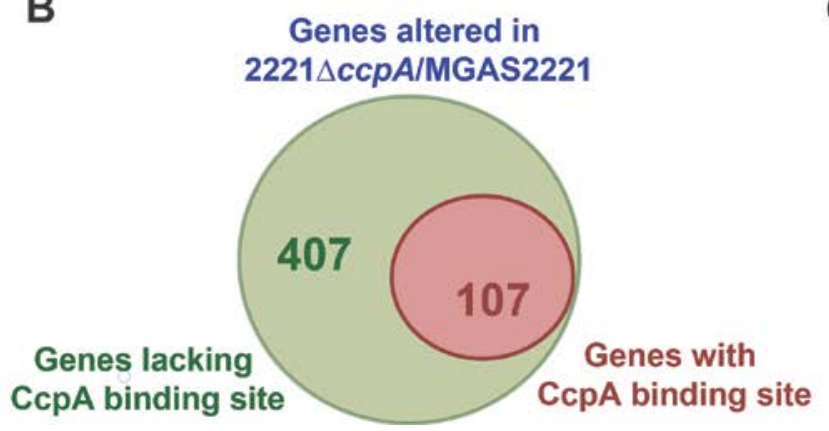

C

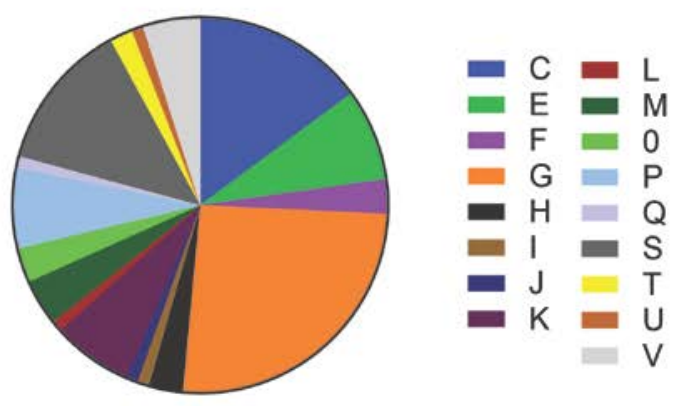

Figure 4. Characterization of in vivo DNA binding of CcpA. (A) Linear representation of indicated strains showing the CcpA binding sites as determined by ChIPseq analysis. (B) Venn diagram showing proportion of genes identified as CcpA-regulated by RNAseq that have CcpA binding sites as identified 
in our ChIPseq analysis. (C) COG distribution of genes that have CcpA binding sites and are transcriptionally altered in $2221 \triangle c c p A$. Description of COG categories is provided in legend of Figure 3B.

Specific CcpA binding was identified for genes encoding four PTS systems known and putatively involved in the transport and utilization of maltose, cellobiose (two operons), and mannose, which stands in contrast to the 13 PTS gene/operons whose transcript levels were affected by CcpA inactivation. We also observed CcpA binding sites in genes/operons encoding three ABC carbohydrate transport systems for maltose, maltodextrin, and sialic acid. The central role of maltose/maltodextrin utilization in GAS pathophysiology is reflected by the five distinct CcpA binding sites for genes involved in the acquisition of these prevalent carbohydrates including that in the promoter region of the pulA gene encoding a cellsurface pullulanase important for both nutrient acquisition and adherence (Figure 5A). In addition to transporters, genes encoding glycerol kinase, phosphoglucomutase, and HPr, which are also involved in carbohydrate metabolism, had CcpA binding sites and were directly regulated by CcpA. 
A

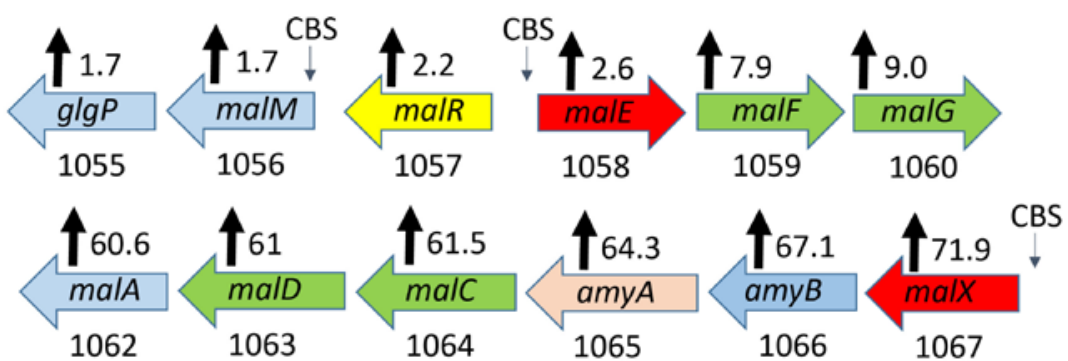

1065 CBS

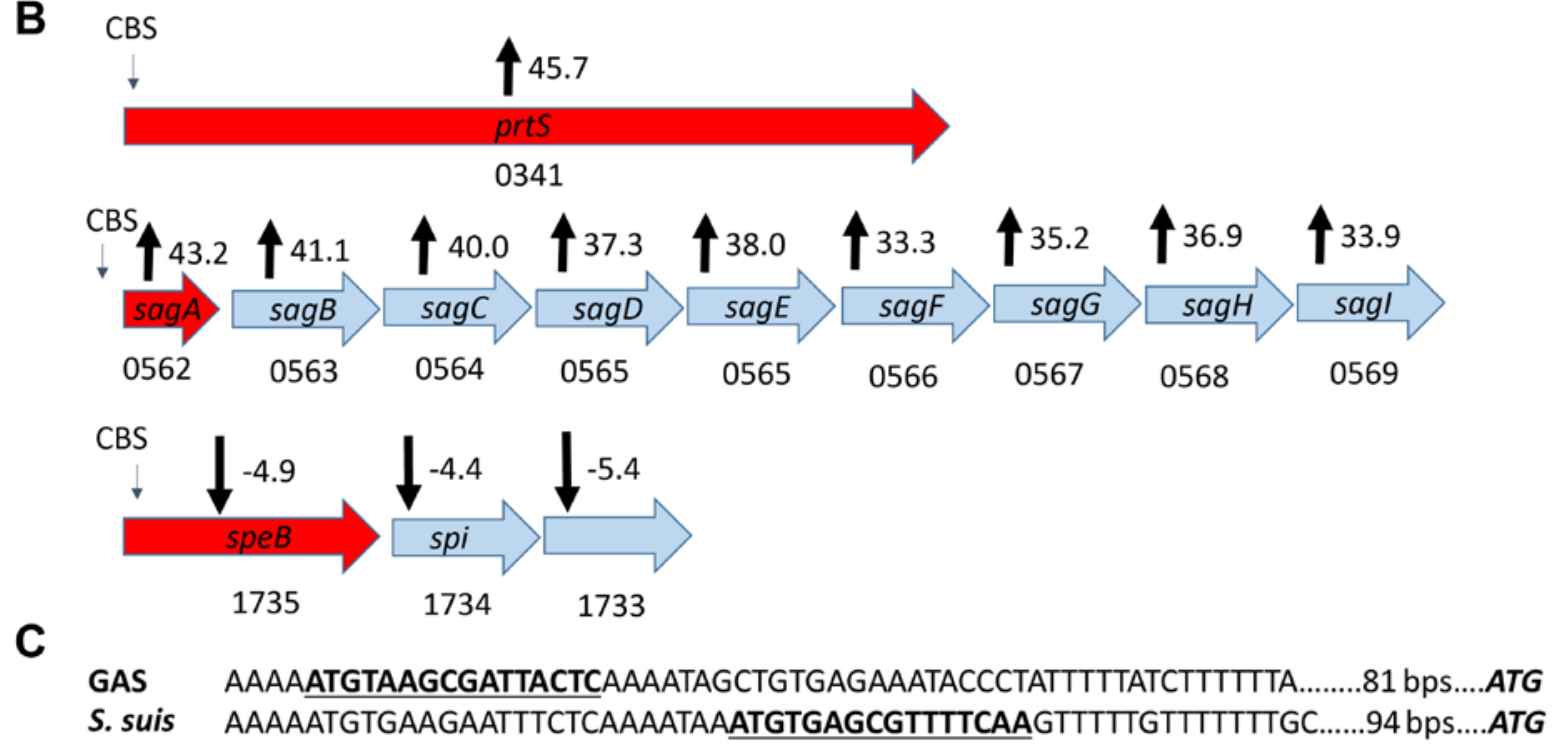

Figure 5. CcpA-bound sites in MGAS2221. Schematic representation of the binding sites for CcpA (CBS) in key (A) carbohydrate transport and (B) virulence factor encoding genes. Fold change in transcript levels upon CcpA inactivation is displayed with black arrows. Positive numbers indicate higher transcript levels in 2221 $\Delta c c p A$ compared to MGAS2221. Genes in the schematic diagram are color coded to indicate function: blue - intracellular carbohydrate processing protein; yellow - transcriptional regulator; red - substrate binding lipoprotein; green - transport protein and beige - cell surface/secreted protein. (C) Sequence variation in the promoter region of the arginine deiminase $(\operatorname{arc} A)$ gene in GAS and S. suis. Enriched site for GAS and putative CcpA binding site for S. suis are highlighted. 
CcpA enriched sites were also identified in the promoters of genes encoding various amino acid uptake and utilization pathways such as those involved in arginine, histidine, and glutamate metabolism. Although a recent CcpA study in Streptococcus suis did not identify in vivo CcpA binding for arcA, the initial gene in the arginine catabolism operon, $\operatorname{arcA}$ show ed the strongest CcpA-mediated DNA enrichment for the entire MGAS2221 dataset. A comparison of the $\operatorname{arcA}$ promoters showed that whereas a CcpA binding site is present in GAS and predicted for $S$. suis, these sites are quite variable both in terms of composition and location, which may explain the divergent results (Figure 5C).

Importantly, DNA enrichment was observed for the promoter and/or 5' coding regions of three key GAS virulence factor encoding genes (Figure 5B). Namely we observed in vivo binding of CcpA for $s a g A$, the first gene in the operon encoding the key cytotoxin Streptolysin $\mathrm{S}, \mathrm{speB}$, which encodes an actively secreted broad-spectrum cysteine proteinase, and prtS which encodes an IL-8 degrading enzyme. No CcpA binding or effect on gene transcript level was observed for cfa (M5005_spy0981) which was previously reported to be directly regulated by CcpA (Kietzman \& Caparon, 2009). Similarly, we did not observe enrichment for the other nine virulence factor encoding genes whose transcript levels were varied by CcpA inactivation (Table 2) suggesting an indirect mechanism for CcpA impact on these genes.

Given the large numbers of genes impacted by CcpA inactivation relative to the number of identified CcpA binding sites, it was reasonable to suspect that many of the genes might be secondarily affected by regulators under CcpA control. Indeed, RNAseq analysis show ed that the transcript levels of 21 genes/operons encoding known and putative transcriptional regulators, including four two-component gene regulatory systems (TCS), were signific antly affected by CcpA inactivation (Supplementary Table 1). Of these, however, we identified direct CcpA binding for only three genes encoding the transcriptional regulators BglG, ArgR2 and Spy1495 (Table 4). While the bglG and $\operatorname{argR2}$ genes had a CcpA-binding site in their promoters, spy1495 was in an operon where the first gene (spy1496) was CcpA-bound. As previously noted, there was also CcpA enrichment in the intergenic region between malE and malR (Figure 5A), which encodes a maltose regulatory protein. Hence, malR could also be 
directly regulated by CcpA although the location of the binding site suggests that CcpA is more likely to influence malE transcription. There was no DNA enrichment for any TCS or the master regulator Mga (multi gene activator), which was identified to be directly regulated by CcpA in a previous study (Almengor et al., 2007). Given that none of the regulators directly controlled by CcpA are known or expected to have large transcriptomes (Shelburne et al., 2011), these data suggest that the broad impact of CcpA inactivation on GAS gene transcript levels is unlikely to be primarily mediated by regulators directly under CcpA control.

\section{Analysis of CcpAV301A in vivo DNA Binding}

Of the 76 DNA loci bound by CcpA, 37 enriched regions which encompass 75 genes (12\% of the CcpA transcriptome) were also enriched in strain 2221-CcpA-V301A (Figure 4A). All of the enriched sites in 2221-CcpA-V301 were associated with genes that also evidenced enriched sites in MGAS2221. All three of the virulence factor encoding genes bound by CcpA in strain MG22221, prtS, sagA, and speB, also evidenced binding in strain 2221-CcpA-V301A (Table 2 and 4). Similarly, five of the seven PTS/ABC carbohydrate transport encoding genes/operons (Table 3 and 4) bound by CcpA were also bound by CcpAV301A as was $\operatorname{arcA}$ (Table 5). In contrast, spy1746, which encodes the first gene in a cellobiose PTS operon, and spy0212, which encodes the first gene in the sialic acid uptake ABC operon were not bound by CcpAV301A. We also did not observe binding of the CcpAV301A protein to the promoter of either ccpA, ptsH (HPr) or the operon encoding the ATP synthase genes (Table 4). When considering all DNA binding sites for the CcpA protein in strain MGAS2221, those sites that were also bound in strain 2221-CcpA-V301A were more likely to have significantly different transcript levels following CcpA inactivation ( $\mathrm{P}<0.001$ by by Fisher's exact test).

\section{Characterization of CcpA binding sites not associated with differentially expressed genes}

For 29 CcpA binding sites, we did not observe a significant difference in transcript levels for a nearby gene following CcpA inactivation. The majority of these binding sites were located in the middle 
of genes suggesting that the observed CcpA binding is likely non-functional. For nine genes, the DNA enrichment overlapped with promoter regions including four genes whose function would suggest regulation by CcpA (Table 4). These four genes were pgi, which encodes a glucose utilization protein, plr, which encodes an aldehyde dehydrogenase, $l d h$, which encodes an enzyme that converts lactate to pyurvate, and the previously mentioned pulA. Of these 29 CcpA enriched sites that were not associated with transcript level variation after CcpA inactivation, 11 were also enriched in strain 2221-CcpA-V301 including three genes encoding carbohydrate utilization proteins (pgi, plr, and pulA) as well as one site in a CRISPR operon and the salY gene, which encodes an $\mathrm{ABC}$ transport protein involved in lantibiotic secretion (Table 4). The combination of CcpA binding site location and putative function suggests that these genes may be directly regulated by CcpA under conditions distinct from those studied herein. 
Table 4. CcpA binding sites identified by ChIPseq analysis and associated genes.

Bound Bound by

V301A cre cre location ${ }^{b}$

cre sequence

Locus tag ${ }^{\mathrm{c}}$

Gene

Regulated

HPr-

\begin{tabular}{|c|c|c|c|c|c|c|c|c|c|c|c|}
\hline 1 & Yes & Yes & Yes & Prom & AAAGAAAGCGGTTTCA & M5005_Spy_0039 & $\begin{array}{l}\operatorname{adh} 2 \\
\operatorname{ack} A\end{array}$ & bifunctional acetaldehyde-CoA/alcohol dehydrogenase & C & Yes & Semi dependent \\
\hline 2 & Yes & Yes & & Prom & ACAGAAAACGATTTCA & & ackA & & & & \\
\hline 3 & Yes & No & Yes & ORF & ATGGTGGCGTTTTCTT & M5005_Spy_0128 & $n t p E$ & V-type ATP synthase subunit E & $\mathrm{C}$ & Yes & Independent \\
\hline 4 & Yes & Yes & Yes & Prom & AACGAAAACCTTTTCA & M5005_Spy_0180 & - & hypothetical protein & $\mathrm{D}$ & No & NA \\
\hline 4 & Yes & Yes & Yes & Prom & AACGAAAACCTTTTCA & M5005_Spy_0185 & $p g i$ & pgi & G & No & NA \\
\hline 5 & Yes & No & Yes & Prom & TTGAAAGCGCTTTATT & M5005_Spy_0212 & - & $\mathrm{N}$-acetylmannosamine-6-phosphate 2-epimerase & G & Yes & Independent \\
\hline 6 & Yes & Yes & Yes & Prom & AAAGAAAGCССТTTCC & M5005_Spy_0233 & $p l r$ & aldehyde dehydrogenase & G & No & NA \\
\hline 7 & Yes & Yes & Yes & Prom & AATGTAAGCGCTAACAAAAT & M5005_Spy_0339 & exoA & exodeoxyribonuclease & $\mathrm{L}$ & Yes & Independent \\
\hline 7 & Yes & Yes & Yes & Prom & AATGTAAGCGCTAACAAAAT & M5005_Spy_0340 & lctO & L-lactate oxidase & $\mathrm{C}$ & Yes & Semi dependent \\
\hline 8 & Yes & Yes & Yes & ORF & TAGGAAGCGTTTTCTT & M5005_Spy_0341 & prtS & peptidase S8 & $\mathrm{O}$ & Yes & Independent \\
\hline 9 & Yes & No & Yes & ORF & GTGCAAGCGCTTTGAT & M5005_Spy_0362 & gcaD & glucosamine-1-phosphate acetyltransferase & M & No & NA \\
\hline 10 & Yes & No & No & - & - & M5005_Spy_0416 & & glutamine cyclotransferase & $\mathrm{O}$ & No & NA \\
\hline 10 & Yes & No & No & - & - & M5005_Spy_0417 & pсp & pyrrolidone-carboxylate peptidase & $\mathrm{O}$ & No & NA \\
\hline 11 & Yes & No & No & - & - & M5005_Spy_0417 & pсp & pyrrolidone-carboxylate peptidase & $\mathrm{O}$ & No & NA \\
\hline 12 & Yes & No & Yes & Prom & TTGAAAACTTTTTCAA & M5005_Spy_0424 & ссрA & catabolite control protein A & $\mathrm{K}$ & Yes & Independent \\
\hline 13 & Yes & No & No & - & - & M5005_Spy_0495 & lys & lysine--tRNA ligase & $\mathrm{J}$ & No & NA \\
\hline 13 & Yes & No & No & - & - & M5005_Spy_0496 & & haloacid dehalogenase & $\mathrm{s}$ & No & NA \\
\hline 14 & Yes & No & No & - & - & M5005_Spy_0504 & pepF & oligoendopeptidase $\mathrm{F}$ & E & No & NA \\
\hline 15 & Yes & Yes & Yes & ORF & TTTGGGAACGATTTCTCAAG & M5005_Spy_0771 & - & CRISP R-associated endonuclease Cas2 & $\mathrm{L}$ & No & NA \\
\hline 16 & Yes & Yes & Yes & Prom & AAATAAAGCGCTTACT & M5005_Spy_0505 & $p p c$ & phosphoenolpyruvate carboxylase & $\mathrm{H}$ & Yes & Fully dependent \\
\hline 17 & Yes & Yes & Yes & Prom & GAGAAAACGTTTTAGT & M5005_Spy_0512 & - & sugar phosphate phosphatase & $\mathrm{s}$ & Yes & Fully dependent \\
\hline 18 & Yes & No & Yes & Prom & TTGACACCGTTTTCAT & M5005_Spy_0533 & - & hypothetical protein & $\mathrm{s}$ & Yes & Independent \\
\hline 18 & Yes & No & Yes & Prom & TTGACACCGTTTTCAT & M5005_Spy_0534 & - & acetoin reductase & IQ & Yes & Semi dependent \\
\hline 19 & Yes & No & Yes & ORF & GAAGATATCGCTTCTA & M5005_Spy_0556 & eno & phosphopyruvate hydratase & $\mathrm{F}$ & No & NA \\
\hline 20 & Yes & No & Yes & Prom & TATTATATCGATTTC T & M5005_Spy_0555 & - & hypothetical protein & $\mathrm{s}$ & No & NA \\
\hline 20 & Yes & No & Yes & Prom & TATTATATCGATTTC T & M5005_Spy_0556 & eno & phosphopyruvate hydratase & $\mathrm{F}$ & No & NA \\
\hline 21 & Yes & Yes & Yes & Prom & AAGAAAGGGTTTACAT & M5005_Spy_0562 & $\operatorname{sag} A$ & streptolysin S family bacteriocin & & Yes & Semi dependent \\
\hline 22 & Yes & No & Yes & ORF & ATGGAAGCTTTTTCAG & M5005_Spy_0622 & - & alkaline phosphatase & M & No & NA \\
\hline 22 & Yes & No & Yes & ORF & ATGGAAGCTTTTTCAG & M5005_Spy_0625 & aroF & chorismate synthase & E & No & NA \\
\hline 23 & Yes & No & Yes & ORF & GTGAAGGGTTTATCAT & M5005_Spy_0772 & - & type II-A CRISP R-associated protein Csn2 & $\mathrm{s}$ & No & NA \\
\hline 24 & Yes & No & No & - & - & M5005_Spy_0778 & $m s r B$ & peptide-methionine (R)-S-oxide reductase & $\mathrm{O}$ & No & NA \\
\hline 25 & Yes & Yes & Yes & ORF & GAAGATAACGATTTCA & M5005_Spy_0817 & dacA1 & D-alanyl-D-alanine carboxypeptidase & M & No & NA \\
\hline 26 & Yes & No & Yes & ORF & TTGTAAGCGCTACCGA & M5005_Spy_0823 & folQ & dihydroneopterin aldolase & $\mathrm{H}$ & No & NA \\
\hline
\end{tabular}


Bound Bound by

cre location ${ }^{b}$

cre sequence

Locus tag ${ }^{\mathrm{c}}$

Gene

Regulated

HPr-

\begin{tabular}{|c|c|c|c|c|c|c|c|c|c|c|c|}
\hline $\operatorname{CBS}^{a}$ & by WT & V301A & cre & cre location ${ }^{b}$ & cre sequence & Locus $\operatorname{tag}^{c}$ & Gene & Annotation & COG & by СсрA & dependency ${ }^{d}$ \\
\hline 27 & Yes & Yes & Yes & Prom & AAGAAAGGGTTTTCAA & M5005_Spy_0834 & - & alcohol dehydrogenase & $\mathrm{E}$ & Yes & Semi dependent \\
\hline 27 & Yes & Yes & Yes & Prom & AAGAAAGGGTTTTCAA & M5005_Spy_0835 & - & acid phosphatase/phosphotransferase & $\mathrm{s}$ & Yes & Semi dependent \\
\hline 28 & Yes & No & Yes & Prom & ACTGATAACGCTTCCAA & M5005_Spy_0873 & $l d h$ & L-lactate dehydrogenase & $\mathrm{C}$ & No & NA \\
\hline 28 & Yes & No & Yes & Prom & ACTGATAACGCTTCCAA & M5005_Spy_0874 & gyrA & DNA gyrase subunit A & $\mathrm{L}$ & No & NA \\
\hline 29 & Yes & No & No & - & - & M5005_Spy_0925 & $r n h B$ & hypothetical protein & $\mathrm{F}$ & No & NA \\
\hline 30 & Yes & Yes & Yes & Prom & CTTGAAACCGCTTGCT & M5005_Spy_0934 & - & lipoate--protein ligase & $\mathrm{H}$ & Yes & Fully dependent \\
\hline 31 & Yes & No & Yes & Prom & AATGAAAGCGTTTATA & M5005_Spy_0938 & $\operatorname{pgm} A$ & phosphoglucomutase & G & Yes & Semi dependent \\
\hline 32 & Yes & No & No & - & - & M5005_Spy_0938 & pgmA & phosphoglucomutase & G & Yes & Semi dependent \\
\hline 33 & Yes & No & No & - & - & M5005_Spy_0938 & pgmA & phosphoglucomutase & G & Yes & Semi dependent \\
\hline 34 & Yes & Yes & Yes & ORF & TAAGATACCGCTTGCA & M5005_Spy_1055 & $g \lg P$ & maltodextrin phosphorylase & G & Yes & Independent \\
\hline 34 & Yes & Yes & Yes & ORF & TAAGATACCGCTTGCA & M5005_Spy_1058 & male & maltose/maltodextrin-binding protein & G & Yes & Independent \\
\hline 35 & Yes & Yes & Yes & Prom & CTGCAAGCGGTTGCAT & M5005_Spy_1057 & malR & LacI family transcriptional regulator & $\mathrm{K}$ & Yes & Independent \\
\hline 35 & Yes & Yes & Yes & Prom & CTGCAAGCGGTTGCAT & M5005_Spy_1058 & male & maltose/maltodextrin-binding protein & G & Yes & Independent \\
\hline 36 & Yes & Yes & Yes & Prom & ATCGTAATCGCTTTCA & M5005_Spy_1067 & malX & sugar $\mathrm{ABC}$ transporter substrate-binding protein & G & Yes & Semi dependent \\
\hline 37 & Yes & Yes & Yes & Prom & TTAGAAAACGCTTTCT & M5005_Spy_1083 & $b g l G$ & transcription antiterminator BglG & G & Yes & Semi dependent \\
\hline 38 & Yes & Yes & Yes & ORF & CTAAAAGCGTTTTCTC & M5005_Spy_1096 & - & thioesterase & $\mathrm{Q}$ & No & NA \\
\hline 39 & Yes & No & Yes & Prom & CATGATAACCСТTACA & M5005_Spy_1122 & $n r d H$ & NrdH-redoxin & $\mathrm{O}$ & Yes & Independent \\
\hline 39 & Yes & No & Yes & Prom & CATGATAACCСТTACA & M5005_Spy_1121 & ptsH & phosphocarrier protein HPr & G & Yes & NA \\
\hline 40 & Yes & Yes & Yes & Prom & CAAGAAATCGCTTTCT & M5005_Spy_1235 & - & phosphomannomutase & G & Yes & Semi dependent \\
\hline 41 & Yes & No & Yes & ORF & CAGAAAACTCTTTCTT & M5005_Spy_1250 & $f t s A$ & cell division protein FtsA & $\mathrm{D}$ & No & NA \\
\hline 42 & Yes & No & Yes & Prom & ATGGAATCGCTTTCTA & M5005_Spy_1258 & - & hypothetical protein & $\mathrm{S}$ & Yes & Independent \\
\hline 43 & Yes & No & Yes & ORF & ATCGTAAGCGCCTCCA & M5005_Spy_1265 & - & ribose operon repressor & & No & NA \\
\hline 44 & Yes & No & Yes & ORF & GTAAAАTCTTTTTCTG & M5005_Spy_1272 & - & arginine:ornithine antiporter & $\mathrm{s}$ & Yes & Semi dependent \\
\hline 45 & Yes & No & No & - & - & M5005_Spy_1274 & & $\mathrm{N}$-acetyltransferase & $\mathrm{K}$ & Yes & Semi dependent \\
\hline 46 & Yes & No & No & - & - & M5005_Spy_1274 & & $\mathrm{N}$-acetyltransferase & $\mathrm{K}$ & Yes & Semi dependent \\
\hline 47 & Yes & No & No & - & - & M5005_Spy_1274 & & $\mathrm{N}$-acetyltransferase & $\mathrm{K}$ & Yes & Semi dependent \\
\hline 48 & Yes & Yes & Yes & Prom & TGAGTAATCGCTTACA & M5005_Spy_1275 & $\operatorname{arcA}$ & arginine deiminase & E & Yes & Semi dependent \\
\hline 48 & Yes & Yes & Yes & Prom & TGAGTAATCGCTTACA & M5005_Spy_1277 & ahrC.2 & arginine regulator & $\mathrm{K}$ & Yes & Semi dependent \\
\hline 49 & Yes & Yes & Yes & ORF & CTGCAATCGTTTACTT & M5005_Spy_1319 & - & RNA methyltransferase & $\mathrm{J}$ & No & NA \\
\hline 49 & Yes & Yes & Yes & ORF & CTGCAATCGTTTACTT & M5005_Spy_1323 & - & hypothetical protein & $\mathrm{L}$ & No & NA \\
\hline 50 & Yes & Yes & Yes & Prom & CTTGAAGCGCTTACTT & M5005_Spy_1328 & - & YigZ family protein & $\mathrm{s}$ & Yes & Independent \\
\hline 50 & Yes & Yes & Yes & Prom & СTTGAAGCGCTTACTT & M5005_Spy_1325 & - & ribosome-associated factor $\mathrm{Y}$ & $\mathrm{J}$ & Yes & Fully dependent \\
\hline 51 & Yes & No & No & - & - & M5005_Spy_1366 & & penicillin-binding protein $2 \mathrm{X}$ & M & No & NA \\
\hline 52 & Yes & Yes & Yes & ORF & GAGAAAAGGATTTCAT & M5005_Spy_1367 & $f t s L$ & cell division protein FtsL & $\mathrm{D}$ & No & NA \\
\hline
\end{tabular}


Bound Bound by

\begin{tabular}{|c|c|c|c|c|c|c|c|c|c|c|c|}
\hline $\mathrm{CBS}^{\mathrm{a}}$ & $\begin{array}{l}\text { Bound } \\
\text { by WT }\end{array}$ & $\begin{array}{c}\text { Bound by } \\
\text { V301A }\end{array}$ & cre & cre location ${ }^{b}$ & cre sequence & Locus tag ${ }^{c}$ & Gene & Annotation & COG & $\begin{array}{l}\text { Regulated } \\
\text { by CcpA }\end{array}$ & $\begin{array}{c}\text { HPr- } \\
\text { dependency d }\end{array}$ \\
\hline 53 & Yes & Yes & Yes & Prom & AAGTAAGCGTTTTCCT & M5005_Spy_1381 & glpK & glycerol kinase & $\mathrm{F}$ & Yes & Independent \\
\hline 54 & Yes & No & No & - & - & M5005_Spy_1382 & & hypothetical protein & $\mathrm{T}$ & Yes & Independent \\
\hline 55 & Yes & Yes & Yes & Prom & CTGTAAGCGATTACTT & M5005_Spy_1387 & - & 2,5-diketo-D-gluconic acid reductase & $\mathrm{C}$ & Yes & Independent \\
\hline 56 & Yes & No & No & - & - & M5005_Spy_1448 & & nuclease & $\mathrm{S}$ & No & NA \\
\hline 57 & Yes & Yes & Yes & Prom & GTGAAAACGTTTTAAA & M5005_Spy_1477 & - & NCS2 family permease & $\mathrm{s}$ & Yes & Semi dependent \\
\hline 57 & Yes & Yes & Yes & Prom & GTGAAAACGTTTTAAA & M5005_Spy_1479 & $\operatorname{manL}$ & PTS mannose transporter subunit EIIAB & G & Yes & Semi dependent \\
\hline 58 & Yes & Yes & Yes & Prom & AAAGAAAACGTTTTCT & M5005_Spy_1496 & phaB & enoyl-CoA hydratase & I & Yes & Independent \\
\hline 59 & Yes & No & No & - & - & M5005_Spy_1497 & dnaJ & chaperone DnaJ & $\mathrm{O}$ & Yes & Fully dependent \\
\hline 60 & Yes & No & Yes & Prom & AAAGAAAACACTTGCA & M5005_Spy_1503 & - & histidine phosphatase family protein & G & Yes & Independent \\
\hline 61 & Yes & No & No & - & - & M5005_Spy_1513 & & aminotransferase & $\mathrm{E}$ & No & NA \\
\hline 61 & Yes & No & No & - & - & M5005_Spy_1514 & & universal stress protein UspA & $\mathrm{T}$ & No & NA \\
\hline 62 & Yes & Yes & Yes & Prom & TGGGAAAACGTTTCCT & M5005_Spy_1569 & $p f l$ & formate acetyltransferase & $\mathrm{C}$ & Yes & Independent \\
\hline 63 & Yes & No & No & - & - & M5005_Spy_1575 & norA & MFS transporter & EGP & Yes & Fully dependent \\
\hline 64 & Yes & No & Yes & Prom & TTTAAAGCTTTTTAA & M5005_Spy_1599 & $p g k$ & phosphoglycerate kinase & $\mathrm{F}$ & No & NA \\
\hline 65 & Yes & Yes & Yes & Prom & ATAAAAGCGTTATCTC & M5005_Spy_1624 & - & hypothetical protein & & No & NA \\
\hline 66 & Yes & Yes & Yes & ORF & AGAGAAACCGGTACCA & M5005_Spy_1627 & salY & $\mathrm{ABC}$ transporter permease & $\mathrm{V}$ & No & NA \\
\hline 67 & Yes & Yes & Yes & Prom & TGCGCAAGCGCTTGCA & M5005_Spy_1680 & pulA & pullulanase & G & No & NA \\
\hline 68 & Yes & Yes & Yes & Prom & GATGCAATCGCTTGCA & M5005_Spy_1692 & - & PTS maltose & G & Yes & Independent \\
\hline 69 & Yes & Yes & Yes & ORF & GTGATAGCGCTATCTT & M5005_Spy_1734 & speB & streptopain & M & Yes & Independent \\
\hline 69 & Yes & Yes & Yes & ORF & GTGATAGCGCTATCTT & M5005_Spy_1736 & - & hypothetical protein & & Yes & Independent \\
\hline 70 & Yes & No & Yes & Prom & TTGTAATCGTTTACAT & M5005_Spy_1746 & - & PTS cellobiose transporter subunit IIA & G & Yes & Semi dependent \\
\hline 71 & Yes & No & No & - & - & M5005_Spy_1758 & & dipeptidase & M & Yes & Semi dependent \\
\hline 72 & Yes & Yes & Yes & Prom & GTGAAAGCGTTATCGT & M5005_Spy_1758 & - & dipeptidase & M & Yes & Semi dependent \\
\hline 73 & Yes & Yes & Yes & Prom & ATGTAAGCGTTATCTAA & M5005_Spy_1772 & - & glutamate formimidoyltransferase & $\mathrm{E}$ & Yes & Independent \\
\hline 73 & Yes & Yes & Yes & Prom & ATGTAAGCGTTATCTAA & M5005_Spy_1770 & hutI & imidazolonepropionase & $\mathrm{Q}$ & Yes & Semi dependent \\
\hline 74 & Yes & Yes & Yes & Prom & CATGAAAACGCCTCCA & M5005_Spy_1779 & rps $B$ & ATP-binding protein & $\mathrm{T}$ & Yes & Semi dependent \\
\hline 75 & Yes & No & No & - & - & M5005_Spy_1807 & $\operatorname{argR2}$ & arginine repressor & $\mathrm{K}$ & Yes & Independent \\
\hline 76 & Yes & Yes & Yes & ORF & ACAGATAACGCTTACT & M5005_Spy_1865 & htrA & serine protease & $\mathrm{O}$ & No & NA \\
\hline
\end{tabular}

a. CcpA binding site number

b. Prom - binding site located in non-coding promoter region upstream of ATG. ORF - binding site identified within coding region ofgene.

c. Locus tag numbers are based on the MGAS5005 genome.

d. NA - not applicable 


\section{Identification of CcpA binding motif}

Using the ChIPseq data, we identified a single consensus motif in the enriched sites for MGAS2221 (Figure 6B) which was highly consistent with previously identified cre motifs from Bacillus species (Marciniak et al., 2012, Schumacher et al., 2004). This motif was present in 57 of the 76 CcpA enriched sites (75\%). For the remaining 19 sites, no particular consensus motif was identified. We did not identify the cre2 sequence of $S$. suis or the flexible binding site of Clostridium acetobutylicum that were recently described (Willenborg et al., 2014, Yang et al., 2017). Compared to enriched areas with cre sites, enriched areas lacking cre sites were less likely to be in genes/gene promoters whose transcript levels were significantly affected by CcpA inactivation ( $\mathrm{P}=0.01$ by Fisher's exact test), suggesting that the cre-containing enriched DNA sites were more biologically impactful under the studied conditions. The cre sites that were associated with genes whose transcript levels were affected by CcpA inactivation, were significantly more likely to be located within 500nt upstream and 50nt downstream of the start codon compared to cre sites that were near genes not impacted by CcpA inactivation $(\mathrm{P}<0.001$ by Fisher's exact test) (Figure 6A). Relative to our previously described in silico derived cre (Figure 6E, (DebRoy et al., 2016)), the major difference for our ChIPseq derived cre was a lack of predominance of $\mathrm{T}$ at positions 1 and 2 (Figure 6B). We compared the cre sites that we found using the ChIP method in this study to those that we had previously predicted in MGAS2221 using in silico analysis of RNAseq data (DebRoy et al., 2016). Both studies were conducted on mid-exponential cells grown in THY. We had predicted 72 cre sites in silico compared to the 57 we found by ChIPseq in this study. Of these sites, only 29 were in common with 20 being transcriptionally altered upon CcpA inactivation in both studies. Importantly, the ChIPseq analysis enabled identific ation of 14 additional binding sites that are near genes that have altered transcript levels in a $\triangle c c p A$ mutant but were not predicted by the in silico approach. These include the cre sites identified in the promoter of prtS, sagA and the three cre sites in the PTS and $\mathrm{ABC}$ transport systems for maltose/maltodextrin. Conversely, the in silico method predicted 23 DNA loci that were transcriptionally impacted by CcpA inactivation in our study but were not bound by CcpA 
in our ChIPseq analysis. These genes included those encoding the transcriptional regulator FruR, an alcohol dehydrogenase, and the ABC transporter Spy1310.

When considering sites enriched in 2221-CcpA-V301A only (Figure 6C), we identified a motif that was highly similar to that for strain MGAS2221. The occurrence of an A at position 15 was reduced in the cre consensus derived from DNA loci in strain 2221-CcpA-V301A compared to that derived from MGAS2221. Of 57 cre sites in MGAS2221, 37 were bound in strain 2221-CcpA-V301, and we did not identify any enriched cre containing sites that were present in strain 2221-CcpA-V301 but not in MGAS2221. For cre sites that were bound only in strain MGAS2221 but not in the strain 2221-CcpAV301A (Figure 6D), the occurrence of a T at position 2 was absolute and markedly increased relative to all cre sites in MGAS2221. Thus, the ChIPseq data show that although GAS cre sites have a similar architecture to other Gram-positive bacteria, in silico prediction of GAS CcpA binding using motifs is problematic. 


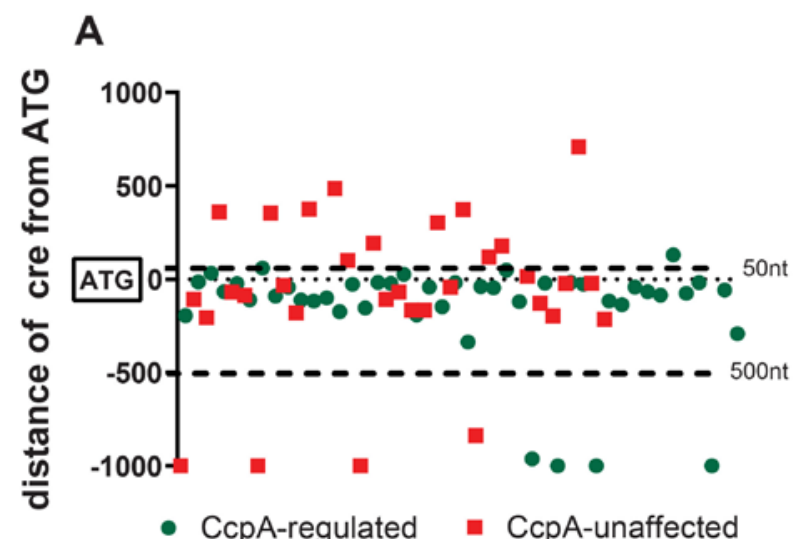

B

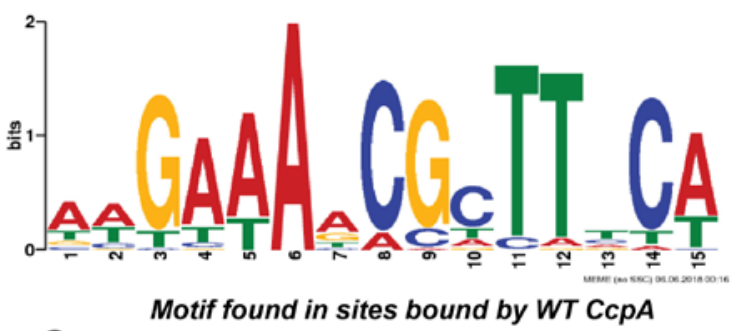

C

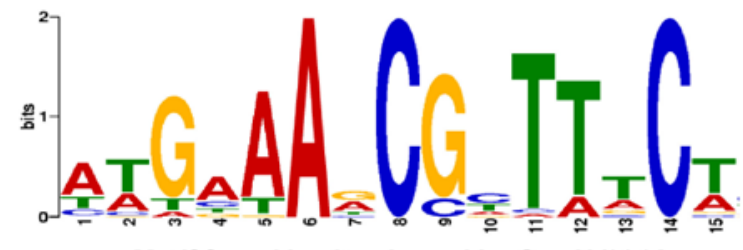

Motif found in sites bound by CcpAV301A

D

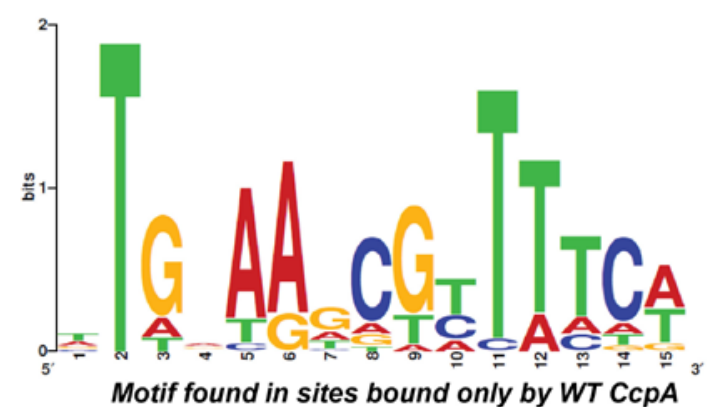

E

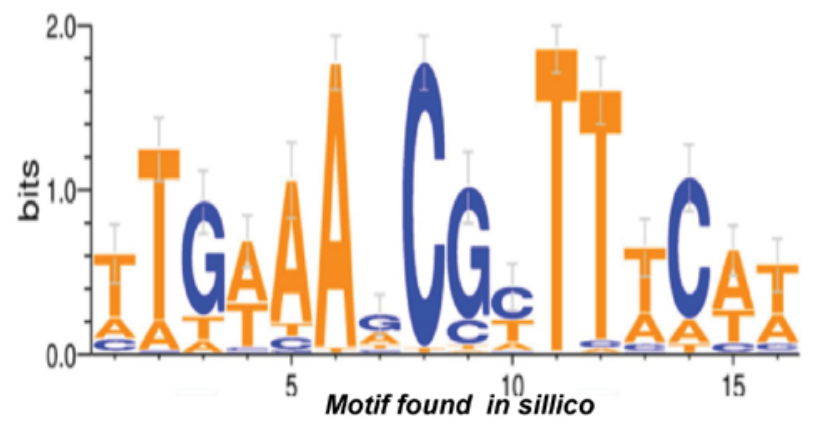


Figure 6. Consensus motifs identified in CcpA binding sites. (A) Scatter plot showing the distance from the translational start site (TSS) of enriched sites bound by the wild type CcpA protein that contain a consensus motif. Genes associated with the enriched sites are color coded to indicate whether they are (CcpA-regulated) or not (CcpA-unaffected) transcriptionally impacted upon CcpA inactivation. Enriched sites that are located farther than 1000nt from the TSS are not plotted. Weblogo representation of the consensus cre motif identified from CcpA binding sites in strains (B) MGAS2221 and (C) 2221-CcpAV301A. (D) The cre motif found in sites that were bound by the wild type CcpA but not the CcpAV301A mutant. (E) The consensus motif identified in our previous study by in sillico analysis of CcpA-regulated genes in three different GAS serotypes (DebRoy et al., 2016).

Impact of CcpAV301A mutation on magnitude of transcript level variation and degree of DNA binding Given our finding of the differential transcript level impact of the CcpAV301A change (Figure 2D), we sought to further study how interruption of CcpA-HPr interaction impacted CcpA function by assessing whether the CcpAV301A amino acid variation quantitatively impacted CcpA gene regulation and DNA binding. For clarity of analysis, we only examined genes whose transcript levels were significantly increased by CcpA inactivation in MGAS2221 (e.g. were CcpA repressed), that evidenced in vivo DNA binding by CcpA, and were monocistronic or the first gene in an operon. We identified 33 genes that met these criteria, all but one of which ( $\operatorname{argR2}$ ) was associated with a cre (Supplementary Table 2). Using these genes, we tested the hypothesis that the CcpAV301A polymorphism w ould result in less release of catabolite repression compared with total CcpA inactivation. Indeed, for 27/33 genes, transcript levels were significantly higher in strain $2221 \Delta c c p A$ relative to 2221-CcpA-V301A, up to 57fold but with a broad range (Supplementary Table 2).

Next, we sought to determine whether this differential impact on transcript levels correlated with a change in DNA binding affinity as determined by amount of DNA immunoprecipitated using anti-CcpA antibody. We used SYBR qPCR to quantify precipitated DNA from the promoter regions of six genes 
that evidenced CcpA binding for both MGAS2221 and 2221-V301A-CcpA strains by ChIPseq. For all six genes, we observed significantly more DNA precipitation in MGAS2221 compared to 2221-CcpAV301A and compared to strains 2221 $\Delta c c p A$ (Figure 7A). How ever, we did not identify a consistent relationship between quantitative differences in amount of DNA precipitated and gene transcript level variation observed in our RNAseq results (Figure 7B). For example, there was markedly more DNA from the ppc and IplA promoters precipitated in strain MGAS2221 compared to 2221-CcpA-V301A(Figure 7A), yet there was no significant difference in $p p c$ and lplA gene transcript levels between strains 2221 and 2221-CcpA-V301A relative to $2221 \Delta c c p A$ (Figure 7B). Conversely, there was only a modest difference in the amount of malX promoter DNA precipitated from MGAS2221 compared to 2221-CcpAV301A (Figure 7A), yet we observed marked variation in transcript levels between MGAS2221 and 2221-CcpA-V301A relative to 2221 $\Delta c c p A$ (Figure 7B). Taken together, we conclude that abrogation of CcpA-HPr interaction quantitatively impacts CcpA function both in terms of the effect of CcpA on gene transcript levels and DNA binding affinity, but decreases in CcpA affinity for promoter DNA due to the V301A alteration does not strictly correlate with a subsequent impact on gene transcript level under the studied conditions. 


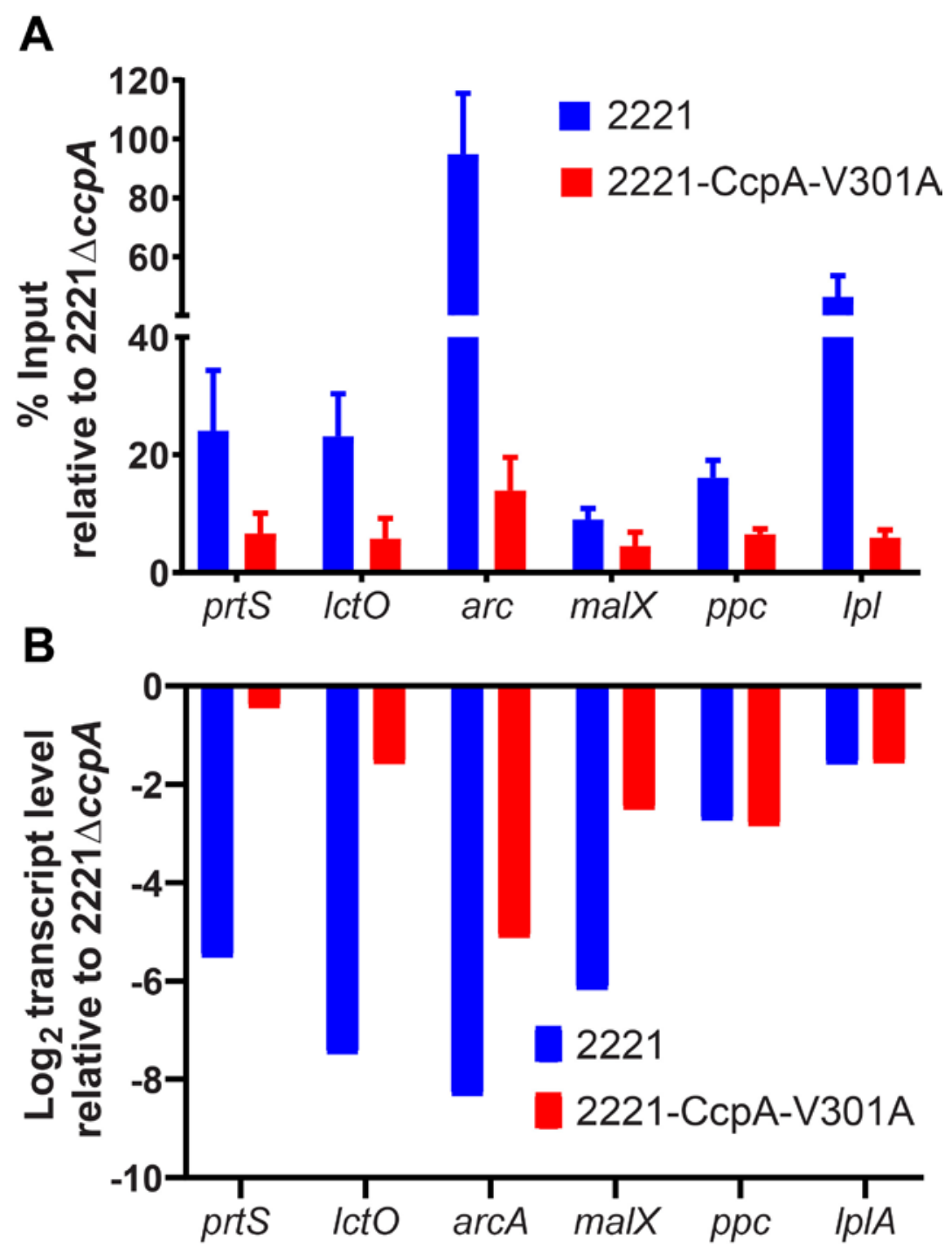

Figure 7. Quantitative impact of CcpAV301A alteration on gene transcript level and DNA binding.

(A) SYBR qRT PCR analysis of DNA precipitated using anti-CcpA antibody from indicated strains (legend inset) for specific promoters (indic ated on x-axis). (B) Transcript level changes as reported in our RNAseq data for the six genes in panel A. 


\section{$\underline{\text { Discussion }}$}

The complex interplay between basic metabolic processes and bacterial pathophysiology is being increasingly appreciated (Eisenreich et al., 2010, Pacheco et al., 2012, Rohmer et al., 2011). For Grampositive bacteria, CcpA stands squarely at this interface given its central role in controlling preferred carbon utilization pathways and regulating virulence factors in a diverse array of human pathogens (Iyer et al., 2005, Mendez et al., 2012, Shelburne et al., 2008, Vega et al., 2016). The primary link of CcpA to the energy status of the bacterial cell is via its interaction with HPr, but study of HPr in many important Gram-positive bacteria has been limited due to its essential nature (Fleming et al., 2015, Willenborg et al., 2014). Herein we sought to extend our knowledge about how CcpA links metabolic and virulence processes through a comprehensive identification of in vivo CcpA binding sites in the major human pathogen group A Streptococcus. Additionally, we created a strain in which CcpA cannot interact with $\mathrm{HPr}$, to delineate HPr-dependent and HPr-independent aspects of CcpA function. Our findings show that CcpA directly regulates the genes encoding several critical GAS virulence factors and that $>50 \%$ of genes in the GAS CcpA transcriptome are impacted by CcpA independent of HPr.

A key contribution of our study is the first genome wide analysis of in vivo CcpA DNA binding for a $\beta$-hemolytic streptococci. We identified 76 CcpA binding sites in GAS, the specificity of which were demonstrated by the lack of enrichment in a CcpA knockout strain. The only other available CcpA ChIPseq analysis in streptococci was performed in Streptococcus suis (Willenborg et al., 2014) and identified 58 DNA loci bound by CcpA at the mid-exponential phase of growth as was studied herein. Some loci, such as those in the promoters of $g l p K, \operatorname{malX}$, and $\mathrm{pgm} A$ were identified in both studies, while others like $\operatorname{arcA}$, manLMN, pgi and eno were not. In MGAS2221 we found the arcA site to be the most strongly enriched peak and the most derepressed gene upon ccpA deletion. In contrast, the $S$. suis study did not identify enrichment of the $\operatorname{arc} A$ or the $\operatorname{man} L M N$ promoters even though these genes were impacted by CcpA inactivation. Additionally, we did not identify the cre 2 motif present in the $S$. suis dataset, perhaps because that motif was identified at the stationary phase of growth, which we did not investigate. 
A common observation of both this and the S. suis study (Willenborg et al., 2014), and of CcpA investigations in other pathogens (Antunes et al., 2012), is that direct CcpA binding accounts for only a small percentage of genes affected by CcpA inactivation. For example, of the genes encoding the 14 known carbohydrate PTS systems in MGAS2221, the transcript levels of 13 were affected by CcpA inactivation, but only four are directly regulated. This narrow direct impact of CcpA on the PTS systems was also observed in S. suis, where CcpA directly impacted only two of the 14 PTS systems present. The manLMN operon in GAS and Streptococcus pneumoniae has been shown to impact a diverse array of other carbohydrate transporters (Abranches et al., 2003, Abranches et al., 2006, Fleming \& Camilli, 2016, Vadeboncoeur \& Pelletier, 1997). We hererin established that in GAS, CcpA binds the promoter of the manLMN operon in vivo (Table 3) and thus could indirectly account for some of the broad impact of CcpA inactivation on carbohydrate transport systems despite only directly binding a small number of genes encoding these systems. While it has been postulated that the broad CcpA transcriptome may be due to the impact of CcpA on other regulators (Antunes et al., 2012, Carvalho et al., 2011, DebRoy et al., 2016, Seidl et al., 2009), we found that CcpA only bound the promoters of a small number of other regulator encoding genes (Table 4) which is not consistent with a broad effect of CcpA on the GAS regulatory netw ork. The marked increase in $\mathrm{HPr} \sim \mathrm{P}$ observed following CcpA inactivation may account for a substantial proportion of the indirect effects of CcpA given the known role of HPr P in a broad array of regulatory processes (Deutscher et al., 2005).

Another key finding of this work was our identification that CcpA directly binds to genes encoding three critical GAS virulence factors, sagA, prtS, and speB. How CcpA mechanistically impacts the expression of the sag operon, and subsequent production of the key cytotoxin streptolysin S, has been an object of study for the past 12 years since the original observation that CcpA inactivation strikingly increases steptolysin S production (Kinkel \& McIver, 2008, Shelburne et al., 2008). Our group as well as others have identified in vitro binding of CcpA to the sagA promoter (Kinkel \& McIver, 2008, Shelburne et al., 2008) whereas an in vivo study showed no precipitation of the sagA promoter using a CcpA antibody (Kietzman \& Caparon, 2009). Although both MGAS2221 and HSC5, the strain used in the 
aforementioned study (Kietzman \& Caparon, 2009), both contain the sagA cre site we identified, the area upstream of the cre site is quite divergent for the two strains which could explain the differential in vivo binding results. It has been shown that the manLMN operon and the $\beta$-glucoside PTS (Table 3), which we identified herein as being directly and indirectly regulated by CcpA respectively, impacts streptolysin production (Braza et al., 2020, Sundar et al., 2017) suggesting that CcpA may regulate streptolysin S production both directly and indirectly. In concert with our data, CcpA was identified as binding in vivo to the gene encoding suilysin, the pore-forming toxin of S. suis, (Willenborg et al., 2014) and to bind in vitro to the sagA promoter of S. anginosus (Bauer et al., 2018). We identified direct binding of CcpA to speB, in accordance with previous studies that have used DNA pulldown and fluorescence polarization methods to demonstrate this interaction (Kietzman \& Caparon, 2009, Shelburne et al., 2010). Interestingly, speB was one of the very few genes directly activated by CcpA. Additionally, our identification that CcpA directly regulates prtS, which encodes a critical IL-8 degrading enzyme, marks the first identification of in vivo binding of a regulator to this important GAS virulence factor. The evolutionary rationale for having such critical and diverse virulence factor encoding genes under direct control of a global metabolic regulator is not clear, but CcpA has also been shown to directly and indirectly impact the expression of key virulence factors in a range of Gram-positive bacteria from Clostridia to Staphylococci (Abranches et al., 2008, Chiang et al., 2011, Seidl et al., 2006, Varga et al., 2004).

Although HPr has long been recognized as critical to CcpA function, study of HPr in major human pathogens such as staphylococci and streptococci has been limited due to its essential nature, including the critical Ser46 residue (Fleming et al., 2015, Willenborg et al., 2014). We also were unable to affect HPrSer46 P through a mutagenesis approach and thus sought to interrupt CcpA-HPr P interaction by modifying the critical CcpAV301 residue. Surprisingly, although the V301A mutation abrogated CcpA-HPr interaction both in vitro and in vivo, more than half of the genes in the CcpA transcriptome still demonstrated CcpA-based repression in strain 2221-CcpA-V301A, indicating the capacity of CcpA to function independently of HPrSer46 P in GAS. We were able to correlate the 
preserved function of the CcpAV301A protein through ChIPSeq analysis which revealed continued enrichment for numerous DNA promoters in strain 2221-CcpA-V301A. Our identification of CcpA functioning independent of HPr echoes findings from a recent ChIPseq study in S. suis which found that CcpA can bind to promoters of and regulate several genes in both exponential and stationary phase of grow th (Willenborg et al., 2014). Given the absence of HPrSer46 P in the stationary phase, they postulated that the CcpA binding and regulation observed in the stationary phase probably occurred independent of HPr (Willenborg et al., 2014). These findings stand in contrast to those from Staphylococcus xylolus in which HPrSer46 P is absolutely essential for CcpA-mediated catabolite repression (Jankovic \& Bruckner, 2002). Our findings are more in concert with studies in B. subtilis, the non-pathogenic gram positive model bacterium, in which the impact of a HPrS46A mutation is essentially an overall dampening of gene regulation in terms of magnitude (Lorca et al., 2005). Indeed, our ChIPseq analysis revealed that enrichment of promoter DNA was typically reduced in strain 2221-CcpA-V301A compared to the wild-type even when significant changes in transcript levels were identified, suggesting that the CcpAV301A bound to cre sites with lower affinity. Similarly, we found that the magnitude of effect on gene transcript levels due to the inability of the CcpAV301A mutant protein to bind HPrSer46 P varied such that genes could be grouped into classes that are strongly, mildly or not impacted by the abrogation of CcpA-HPr interaction.

One possible purpose of the varied magnitude of effects on gene transcript levels observed in the 2221-CcpA-V301A strain would be to facilitate a broad array of transcriptional responses to carbohydrate availability and intracellular energy status (Paluscio et al., 2018). GAS infects numerous different infection sites such as skin, oropharynx, blood and muscle (Cole et al., 2011, Cunningham, 2000, Walker et al., 2014). Each of these sites have unique nutritional and environmental makeup. The dependence/independence of CcpA from HPr P could assist with fine-tuning regulation of pertinent genes by CcpA such that site specific resources can be utilized optimally to aid the pathogen. For example, a recent study compared the GAS transcriptome during necrotizing fasciitis to growth in standard laboratory medium (Kachroo et al., 2020). Some of the most highly upregulated genes in 
Kachroo et. al,. including sagA, $\operatorname{arcA}$, and the cellobiose PTS operon, were directly bound by CcpA in our study, all of which we classified as HPr-dependent. Conversely, the transcript levels of the directly CcpA bound, HPr-independent genes of the sialic acid operon, prtS, ackA, and hpr showed no significant upregulation during necrotizing fasciitis (Kachroo et al., 2020). These findings are consistent with the concept that CcpA can maintain repression of these genes even under metabolically unfavorable conditions when HPrSer46 P levels would be expected to be low.

In conclusion, herein we present a genome wide analysis of chromatin occupancy by CcpA which reveals that CcpA directly regulates multiple key GAS virulence factors in addition to a broad array of critical metabolic genes. Through use of a CcpA isoform incapable of interacting with HPr, we have delineated not only the role of HPr in CcpA-mediated gene regulation but also the ability of CcpA to function at certain sites independently of its key co-factor. These findings extend the mechanistic understanding of how CcpA contributes to the pathophysiology of Gram-positive bacteria. 


\section{$\underline{\text { Experimental Procedures }}$}

\section{Bacterial strains, media and growth conditions}

All strains used in this study are listed in Table 1. Group A Streptococcus (GAS) strains were routinely grown in Todd-Hewitt media supplemented with $0.2 \%$ yeast extract (THY) at $37^{\circ} \mathrm{C}$ with $5 \% \mathrm{CO}_{2}$. A single amino acid change in cсpA was engineered into the wild type MGAS2221 strain by homologous recombination using the pBBL740 plasmid as described previously (Horstmann et al., 2018), to create the isoallelic strain 2221-CcpA-V301A.

\section{Recombinant proteins and antibodies}

Site directed mutagenesis (primers in Supplementary Table 3) was used to introduce the V301A mutation into an existing clone of GAS ccpA (Shelburne et al., 2008) in the pET-His2 vector to generate the pET-His2-V301A plasmid, which was transformed into E. coli BL21/pLysS. Wild type CcpA and V301A mutant protein were induced overnight at $18^{\circ} \mathrm{C}$, and purified to $>95 \%$ homogeneity as described previously (Shelburne et al., 2010) and extensively buffer exchanged to 20mM Tris/HCl pH 7.5, 200mM $\mathrm{NaCl}$. Recombinant GAS HPr was overexpressed, phosphorylated and purified as described previously (Shelburne et al., 2010). For the generation of polyclonal antibodies purified wild type CcpA and HPr proteins were used to immunize rabbits and affinity-purified antibody was obtained (Covance, Denver, PA).

\section{Protein-Protein interaction analysis}

Surface Plasmon Resonanace (SPR) analysis was performed at $25^{\circ} \mathrm{C}$ using BIAcore T200 instrument (GE Healthcare). Wild type CcpA and V301A mutant proteins were immobilized on sensor chip CM5 via amine coupling. PBS (phosphate buffered saline (8.06 mM Na2HPO4 and 1.94 mM KH2PO4, pH 
experiment. Sensor chip surface was activated by injecting a 1:1 mixture of 0.4 M of 1-ethyl-3-(3dimethylaminopropyl) carbodiimide hydrochloride and $0.1 \mathrm{M}$ of $\mathrm{N}$-hydroxysuccinimide over the flow cell surface at $5 \mu \mathrm{l} / \mathrm{min}$ for 7 minutes. After the surface was activated, CcpA $(10 \mu \mathrm{g} / \mathrm{ml}$ in $10 \mathrm{mM} \mathrm{NaOAc} \mathrm{pH}$ 5.0) and CcpAV301A (20 $\mu \mathrm{g} / \mathrm{ml}$ in 10mM NaOAc pH 5.0) were injected over different flow cells. Sensor surface was deactivated with an injection of $1.0 \mathrm{M}$ ethanolamine- $\mathrm{HCl} \mathrm{pH} 8.5$ at $5 \mu \mathrm{l} / \mathrm{min}$ for 7 minutes. Approximately, 3700 response units (RU) of CcpA and 4200 RU of CcpAV301A were immobilized. A reference flow cell was prepared with activation and deactivation steps but no protein was immobilized. Two-fold dilutions of HPr or HPrSer46 P from $1.25 \mu \mathrm{M}$ to $80 \mu \mathrm{M}$ in PBS were flown over the immobilized proteins at $20 \mu \mathrm{l} / \mathrm{min}$ for 30 seconds. All SPR responses were baseline corrected by subtracting the response generated from the corresponding reference surface. Double-referenced SPR response curves (with the buffer blank run further subtracted) were used for affinity determination. The equilibrium response of each injection was collected and plotted against the concentration of injected protein. A one-site binding (hyperbola) model was fitted to the data (GraphPad Prism 4) to obtain the equilibrium dissociation constant $K_{\mathrm{D}}$.

\section{PhosTag gel}

Recombinant HPr protein was phosphorylated and purified as described previously (Shelburne et al., 2010). Cell lysates of GAS strains were prepared, separated on $12.5 \%$ SuperSep Phostag gels and detected using a polyclonal anti-HPr antibody (Horstmann et al., 2014) as described previously (Horstmann et al., 2018). Experiments were repeated at least twice using samples collected on separate days.

\section{Co-immunnoprecipitation}

Strains MGAS2221, 2221 $\Delta c c p A$, and 2221-CcpA-V301A were grown to mid-exponential phase in THY, crosslinked with EGS [ethylene glycol bis(succinic acid $N$-hydroxysuccinimide ester)] and 
formaldehyde and harvested. Pellets were sonicated and CcpA-containing complexes were immunoprecipitated using a polyclonal anti-CcpA antibody and then analyzed for the presence of HPr by western blotting using a polyclonal anti-HPr antibody and the Odyssey imaging system as described previously (Horstmann et al., 2015).

\section{Analysis of transcript levels}

For RNA seq analysis, RNA was isolated from four replic ate cultures for each strain grown to midexponential phase in THY (OD 0.5) using the RNeasy kit (Qiagen) and processed as previously described (Horstmann et al., 2014). RNAseq data analysis was performed as previously described (Sanson \& Flores, 2020). As the MGAS2221 genome is not publicly available, we used the MGAS5005 genome as the reference genome, which is identical to MGAS2221 (Sumby et al., 2005) in gene content. For Taqman real-time qRT-PCR, strains were grown in duplicate on two separate occasions to midexponential phase in THY and processed as described previously (Horstmann et al., 2014). The gene transcript levels between MGAS2221 and either 2221 $\Delta$ ccpA or 2221-CcpA-V301A was compared using an ordinary one way ANOVA. Primers and probes used are listed in Supplementary Table 3.

\section{Chromatin Immunoprecipitation (ChIP) and sequencing (ChIPseq)}

For ChIP analysis, MGAS2221, 2221 $\Delta c c p A$ and 2221-CcpA-V301A strains were grown to midexponential phase in THY. Crosslinking was performed with $1 \%$ formaldehyde and quenched with 0.125M glycine. DNA was sheared using a Diagenode Bioruptor Plus and immunoprecipitated with antiCcpA antibody. We performed ChIPseq for two independent replicates of each strain. ChIP sequencing was performed in the Advanced Technology Genomics Core (ATGC) at MD Anderson Cancer Center. Briefly, Illumina compatible Indexed libraries were prepared from 12ng of Diagenode Biorupter Pico sheared ChIP DNA using the KAPA Hyper Library Preparation Kit (Roche). Libraries were enriched with 2 cycles of PCR then assessed for size distribution using the 4200 TapeStation High Sensitivity D1000 ScreenTape (Agilent Technologies) and quantified using the Qubit dsDNA HS Assay Kit (Thermo 
Fisher). Equimolar quantities of the indexed libraries were multiplexed, 9 libraries per pool. The pool was quantified by qPCR using the KAPA Library Quantification Kit (Roche) then sequenced on the Illumina NextSeq500 sequencer using the 75nt high-output flow cell. Raw FASTQ files from DNA sequencing were trimmed through Trimmomatic v. 0.36 (Bolger et al., 2014). A sliding window quality trimming was performed, cutting once the average quality of a window of three bases fell $<30$. Reads shorter than 32 bp after trimming were discarded. Resulting data were aligned to Genbank reference genome of Streptococcus pyogenes MGAS5005 (NC_007297.2) using Bowtie2 v. 2.3.0 (Langmead \& Salzberg, 2012), without allowing mismatch. Resulting SAM files were converted to BED format using sam2bed v. 2.4.33. For each sample, the coverage per base was determined using coverageBed v. 2.27.1 from BEDTools (Quinlan \& Hall, 2010). Subsequent processing was done using in-house Perl scripts. For that, data were normalized to the same average read depth and mean coverage per base was determined for each pair of replicates (Sharma et al., 2017). To select peaks for CcpA in MGAS2221 and 2221-CcpA-V301A, we compared the coverage base per base of both conditions with $2221 \Delta c c p A$, and selected those bases scoring $\geq 2.0$ fold over $2221 \Delta c c p A$. Final peaks were obtained by merging selected bases using mergeBed v. 2.27.1. The sequences of assembled peaks were retrieved and submitted to Multiple Em for Motif Elicitation v. 5.0.0 (MEME) (Bailey \& Elkan, 1994, Bailey et al., 2009) for motif searching. The expected number of sites was set to one per sequence and the minimum motif width was set to 15 bp (Sharma et al., 2017). A single statistically significant motif (E-value $<1 \mathrm{e}-12$ ) was recovered for MGAS2221 and 2221-CcpA-V301A and these matched the known CcpA binding consensus (DebRoy et al., 2016). The E-value is derived by MEME, from the motif's log likelihood ratio, taking the motif length and background DNA sequence into account. Manual visualization of identified motifs and closest coding genes (CDS) on S. pyogenes MGAS5005 genome was performed using Integrative Genomic Viewer v. 2.3 (IGV) (Thorvaldsdottir et al., 2013).

\section{Acknowledgements}


This work was supported by RO1 AI089891 (S.A.S) from the National Institues of Health, CONICYT-PIA Program AFB170001 of the Center for Mathematical Modeling (M.L), FONDECYT № 1190742 (M.L), Center for Genome Regulation FONDAP 15090007 (M.L), Mesa Minería Consorcio de Universidades del Estado de Chile - CUECH (M.L) and Fondo de Innovación para la

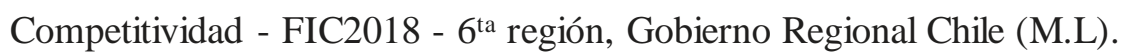

We acknow ledge the CCSG P30 CA016672 funds for the Bioinformatics Shared Resource and the Sequencing and Microarray facility at MD Anderson Cancer Center.

We would like to acknowledge Kunal Rai and Christopher Terranova for their knowledge and guidance in developing ChIPseq methodology for GAS.

\section{Author Contributions}

S.D., S.A.S., V.A., G.G., M.L. and M.H. designed the research, S.D., S.A., N.H. and S.A.S. did the experiments, S.D., X.L., S.A.S., A.R.F, V.A., G.G., V.M., M.L. and M.H. performed data analysis and S.D., X.L., M.L., M.Hand S.A.S. wrote the manuscript. All authors reviewed the manuscript.

\section{Competing Financial Interests}

The authors have no competing financial interests.

\section{$\underline{\text { References }}$}

Abranches, J., Candella, M.M., Wen, Z.T., Baker, H.V., and Burne, R.A. (2006) Different roles of EllABMan and EIIGIc in regulation of energy metabolism, biofilm development, and competence in Streptococcus mutans. J Bacteriol 188: 3748-3756.

Abranches, J., Chen, Y.Y., and Burne, R.A. (2003) Characterization of Streptococcus mutans strains deficient in EIIAB Man of the sugar phosphotransferase system. Appl Environ Microbio/69: 47604769.

Abranches, J., Nascimento, M.M., Zeng, L., Browngardt, C.M., Wen, Z.T., Rivera, M.F., and Burne, R.A. (2008) CcpA regulates central metabolism and virulence gene expression in Streptococcus mutans. J Bacteriol 190: 2340-2349.

Almengor, A.C., Kinkel, T.L., Day, S.J., and Mclver, K.S. (2007) The catabolite control protein CcpA binds to Pmga and influences expression of the virulence regulator Mga in the group A Streptococcus. J Bacteriol 189: 8405-8416. 
Antunes, A., Camiade, E., Monot, M., Courtois, E., Barbut, F., Sernova, N.V., Rodionov, D.A., MartinVerstraete, I., and Dupuy, B. (2012) Global transcriptional control by glucose and carbon regulator CcpA in Clostridium difficile. Nucleic Acids Res 40: 10701-10718.

Aung-Hilbrich, L.M., Seidel, G., Wagner, A., and Hillen, W. (2002) Quantification of the influence of HPrSer46P on CcpA-cre interaction. J Mol Biol 319: 77-85.

Bailey, T.L., Boden, M., Buske, F.A., Frith, M., Grant, C.E., Clementi, L., Ren, J., Li, W.W., and Noble, W.S. (2009) MEME SUITE: tools for motif discovery and searching. Nucleic Acids Res 37: W202-208.

Bailey, T.L., and Elkan, C. (1994) Fitting a mixture model by expectation maximization to discover motifs in biopolymers. Proc Int Conf Intell Syst Mol Biol 2: 28-36.

Bauer, R., Mauerer, S., and Spellerberg, B. (2018) Regulation of the beta-hemolysin gene cluster of Streptococcus anginosus by CcpA. Sci Rep 8: 9028.

Bolger, A.M., Lohse, M., and Usadel, B. (2014) Trimmomatic: a flexible trimmer for Illumina sequence data. Bioinformatics 30: 2114-2120.

Braza, R.E., Silver, A.B., Sundar, G.S., Davis, S.E., Razi, A., Islam, E., Hart, M., Zhu, J., Le Breton, Y., and Mclver, K.S. (2020) PTS Uptake and Metabolism of the ss-Glucoside Salicin Impacts Group A Streptococcal Bloodstream Survival and Soft Tissue Infection. Infect Immun.

Buescher, J.M., Liebermeister, W., Jules, M., Uhr, M., Muntel, J., Botella, E., Hessling, B., Kleijn, R.J., Le Chat, L., Lecointe, F., Mader, U., Nicolas, P., Piersma, S., Rugheimer, F., Becher, D., Bessieres, P., Bidnenko, E., Denham, E.L., Dervyn, E., Devine, K.M., Doherty, G., Drulhe, S., Felicori, L., Fogg, M.J., Goelzer, A., Hansen, A., Harwood, C.R., Hecker, M., Hubner, S., Hultschig, C., Jarmer, H., Klipp, E., Leduc, A., Lewis, P., Molina, F., Noirot, P., Peres, S., Pigeonneau, N., Pohl, S., Rasmussen, S., Rinn, B., Schaffer, M., Schnidder, J., Schwikowski, B., Van Dijl, J. M., Veiga, P., Walsh, S., Wilkinson, A.J., Stelling, J., Aymerich, S., and Sauer, U. (2012) Global network reorganization during dynamic adaptations of Bacillus subtilis metabolism. Science 335: 10991103.

Carvalho, S.M., Kloosterman, T.G., Kuipers, O.P., and Neves, A.R. (2011) CcpA ensures optimal metabolic fitness of Streptococcus pneumoniae. PLoS One 6: e26707.

Chiang, C., Bongiorni, C., and Perego, M. (2011) Glucose-dependent activation of Bacillus anthracis toxin gene expression and virulence requires the carbon catabolite protein CcpA. J Bacterio/ 193: 5262.

Cole, J.N., Barnett, T.C., Nizet, V., and Walker, M.J. (2011) Molecular insight into invasive group A streptococcal disease. Nat Rev Microbiol 9: 724-736.

Cunningham, M.W. (2000) Pathogenesis of group A streptococcal infections. Clin Microbiol Rev 13: 470511.

DebRoy, S., Saldana, M., Travisany, D., Montano, A., Galloway-Pena, J., Horstmann, N., Yao, H., Gonzalez, M., Maass, A., Latorre, M., and Shelburne, S.A. (2016) A Multi-Serotype Approach Clarifies the Catabolite Control Protein A Regulon in the Major Human Pathogen Group A Streptococcus. Sci Rep 6: 32442.

Deutscher, J. (2008) The mechanisms of carbon catabolite repression in bacteria. Curr Opin Microbiol 11: 87-93.

Deutscher, J., Francke, C., and Postma, P.W. (2006) How phosphotransferase system-related protein phosphorylation regulates carbohydrate metabolism in bacteria. Microbiol Mol Biol Rev 70: 9391031.

Deutscher, J., Herro, R., Bourand, A., Mijakovic, I., and Poncet, S. (2005) P-Ser-HPr--a link between carbon metabolism and the virulence of some pathogenic bacteria. Biochim Biophys Acta 1754: 118-125.

Eisenreich, W., Dandekar, T., Heesemann, J., and Goebel, W. (2010) Carbon metabolism of intracellular bacterial pathogens and possible links to virulence. Nat Rev Microbiol 8: 401-412. 
Fleming, E., and Camilli, A. (2016) ManLMN is a glucose transporter and central metabolic regulator in Streptococcus pneumoniae. Mol Microbiol 102: 467-487.

Fleming, E., Lazinski, D.W., and Camilli, A. (2015) Carbon catabolite repression by seryl phosphorylated $\mathrm{HPr}$ is essential to Streptococcus pneumoniae in carbohydrate-rich environments. Mol Microbiol 97: 360-380.

Fujita, Y. (2009) Carbon catabolite control of the metabolic network in Bacillus subtilis. Biosci Biotechnol Biochem 73: 245-259.

Galinier, A., Haiech, J., Kilhoffer, M.C., Jaquinod, M., Stulke, J., Deutscher, J., and Martin-Verstraete, I. (1997) The Bacillus subtilis crh gene encodes a HPr-like protein involved in carbon catabolite repression. Proc Natl Acad Sci U S A 94: 8439-8444.

Giammarinaro, P., and Paton, J.C. (2002) Role of RegM, a homologue of the catabolite repressor protein CcpA, in the virulence of Streptococcus pneumoniae. Infect Immun 70: 5454-5461.

Gorke, B., and Stulke, J. (2008) Carbon catabolite repression in bacteria: many ways to make the most out of nutrients. Nat Rev Microbiol 6: 613-624.

Henkin, T.M., Grundy, F.J., Nicholson, W.L., and Chambliss, G.H. (1991) Catabolite repression of alphaamylase gene expression in Bacillus subtilis involves a trans-acting gene product homologous to the Escherichia coli lacl and galR repressors. Mol Microbiol 5: 575-584.

Homeyer, N., Essigke, T., Meiselbach, H., Ullmann, G.M., and Sticht, H. (2007) Effect of HPr phosphorylation on structure, dynamics, and interactions in the course of transcriptional control. J Mol Model 13: 431-444.

Horstmann, N., Sahasrabhojane, P., Saldana, M., Ajami, N.J., Flores, A.R., Sumby, P., Liu, C.G., Yao, H., Su, X., Thompson, E., and Shelburne, S.A. (2015) Characterization of the effect of the histidine kinase CovS on response regulator phosphorylation in group A Streptococcus. Infect Immun 83: 1068-1077.

Horstmann, N., Saldana, M., Sahasrabhojane, P., Yao, H., Su, X., Thompson, E., Koller, A., and Shelburne, S.A., 3rd (2014) Dual-site phosphorylation of the control of virulence regulator impacts group a streptococcal global gene expression and pathogenesis. PLoS Pathog 10: e1004088.

Horstmann, N., Tran, C.N., Brumlow, C., DebRoy, S., Yao, H., Nogueras Gonzalez, G., Makthal, N., Kumaraswami, M., and Shelburne, S.A. (2018) Phosphatase activity of the control of virulence sensor kinase CovS is critical for the pathogenesis of group A streptococcus. PLoS Pathog 14: e1007354.

Iyer, R., Baliga, N.S., and Camilli, A. (2005) Catabolite control protein A (CcpA) contributes to virulence and regulation of sugar metabolism in Streptococcus pneumoniae. J Bacterio/ 187: 8340-8349.

Jankovic, I., and Bruckner, R. (2002) Carbon catabolite repression by the catabolite control protein CcpA in Staphylococcus xylosus. J Mol Microbiol Biotechnol 4: 309-314.

Johnson, B.P., Jensen, B.J., Ransom, E.M., Heinemann, K.A., Vannatta, K.M., Egland, K.A., and Egland, P.G. (2009) Interspecies signaling between Veillonella atypica and Streptococcus gordonii requires the transcription factor CcpA. J Bacterio/ 191: 5563-5565.

Kachroo, P., Eraso, J.M., Olsen, R.J., Zhu, L., Kubiak, S.L., Pruitt, L., Yerramilli, P., Cantu, C.C., Ojeda Saavedra, M., Pensar, J., Corander, J., Jenkins, L., Kao, L., Granillo, A., Porter, A.R., DeLeo, F.R., and Musser, J.M. (2020) New Pathogenesis Mechanisms and Translational Leads Identified by Multidimensional Analysis of Necrotizing Myositis in Primates. mBio 11.

Kietzman, C.C., and Caparon, M.G. (2009) CcpA and LacD.1 affect temporal regulation of S. pyogenes virulence genes. Infect Immun.

Kietzman, C.C., and Caparon, M.G. (2011) Distinct time-resolved roles for two catabolite-sensing pathways during Streptococcus pyogenes infection. Infect Immun 79: 812-821.

Kinkel, T.L., and Mclver, K.S. (2008) CcpA-mediated repression of streptolysin S expression and virulence in the group A Streptococcus. Infect Immun 76: 3451-3463. 
Langmead, B., and Salzberg, S.L. (2012) Fast gapped-read alignment with Bowtie 2. Nature methods 9: 357-359.

Le Breton, Y., Belew, A.T., Valdes, K.M., Islam, E., Curry, P., Tettelin, H., Shirtliff, M.E., El-Sayed, N.M., and Mclver, K.S. (2015) Essential Genes in the Core Genome of the Human Pathogen Streptococcus pyogenes. Sci Rep 5: 9838.

Leboeuf, C., Leblanc, L., Auffray, Y., and Hartke, A. (2000) Characterization of the ccpA gene of Enterococcus faecalis: identification of starvation-inducible proteins regulated by ccpA. $J$ Bacterio/ 182: 5799-5806.

Lorca, G.L., Chung, Y.J., Barabote, R.D., Weyler, W., Schilling, C.H., and Saier, M.H., Jr. (2005) Catabolite repression and activation in Bacillus subtilis: dependency on CcpA, $\mathrm{HPr}$, and $\mathrm{HprK}$. J Bacteriol 187: 7826-7839.

Ludwig, H., Rebhan, N., Blencke, H.M., Merzbacher, M., and Stulke, J. (2002) Control of the glycolytic gapA operon by the catabolite control protein A in Bacillus subtilis: a novel mechanism of CcpAmediated regulation. Mol Microbiol 45: 543-553.

Marciniak, B.C., Pabijaniak, M., de Jong, A., Duhring, R., Seidel, G., Hillen, W., and Kuipers, O.P. (2012) High- and low-affinity cre boxes for CcpA binding in Bacillus subtilis revealed by genome-wide analysis. BMC Genomics 13: 401.

Mendez, M.B., Goni, A., Ramirez, W., and Grau, R.R. (2012) Sugar inhibits the production of the toxins that trigger clostridial gas gangrene. Microb Pathog 52: 85-91.

Mertins, S., Joseph, B., Goetz, M., Ecke, R., Seidel, G., Sprehe, M., Hillen, W., Goebel, W., and MullerAltrock, S. (2007) Interference of components of the phosphoenolpyruvate phosphotransferase system with the central virulence gene regulator PrfA of Listeria monocytogenes. J Bacteriol 189: 473-490.

Miwa, Y., Nakata, A., Ogiwara, A., Yamamoto, M., and Fujita, Y. (2000) Evaluation and characterization of catabolite-responsive elements (cre) of Bacillus subtilis. Nucleic Acids Res 28: 1206-1210.

Pacheco, A.R., Curtis, M.M., Ritchie, J.M., Munera, D., Waldor, M.K., Moreira, C.G., and Sperandio, V. (2012) Fucose sensing regulates bacterial intestinal colonization. Nature 492: 113-117.

Paluscio, E., Watson, M.E., Jr., and Caparon, M.G. (2018) CcpA Coordinates Growth/Damage Balance for Streptococcus pyogenes Pathogenesis. Sci Rep 8: 14254.

Poncet, S., Mijakovic, I., Nessler, S., Gueguen-Chaignon, V., Chaptal, V., Galinier, A., Boel, G., Maze, A., and Deutscher, J. (2004) HPr kinase/phosphorylase, a Walker motif A-containing bifunctional sensor enzyme controlling catabolite repression in Gram-positive bacteria. Biochim Biophys Acta 1697: 123-135.

Quinlan, A.R., and Hall, I.M. (2010) BEDTools: a flexible suite of utilities for comparing genomic features. Bioinformatics 26: 841-842.

Rohmer, L., Hocquet, D., and Miller, S.I. (2011) Are pathogenic bacteria just looking for food? Metabolism and microbial pathogenesis. Trends Microbiol 19: 341-348.

Sanson, M., and Flores, A.R. (2020) Group A Streptococcus Transcriptome Analysis. Methods Mol Biol 2136: 113-133.

Schumacher, M.A., Allen, G.S., Diel, M., Seidel, G., Hillen, W., and Brennan, R.G. (2004) Structural basis for allosteric control of the transcription regulator CcpA by the phosphoprotein HPr-Ser46-P. Cell 118: 731-741.

Schumacher, M.A., Seidel, G., Hillen, W., and Brennan, R.G. (2006) Phosphoprotein Crh-Ser46-P displays altered binding to CcpA to effect carbon catabolite regulation. J Biol Chem 281: 6793-6800.

Schumacher, M.A., Sprehe, M., Bartholomae, M., Hillen, W., and Brennan, R.G. (2011) Structures of carbon catabolite protein A-(HPr-Ser46-P) bound to diverse catabolite response element sites reveal the basis for high-affinity binding to degenerate DNA operators. Nucleic Acids Res 39 : 2931-2942. 
Seidl, K., Bischoff, M., and Berger-Bachi, B. (2008a) CcpA mediates the catabolite repression of tst in Staphylococcus aureus. Infect Immun 76: 5093-5099.

Seidl, K., Goerke, C., Wolz, C., Mack, D., Berger-Bachi, B., and Bischoff, M. (2008b) Staphylococcus aureus CcpA affects biofilm formation. Infect Immun 76: 2044-2050.

Seidl, K., Muller, S., Francois, P., Kriebitzsch, C., Schrenzel, J., Engelmann, S., Bischoff, M., and BergerBachi, B. (2009) Effect of a glucose impulse on the CcpA regulon in Staphylococcus aureus. BMC Microbiol 9: 95.

Seidl, K., Stucki, M., Ruegg, M., Goerke, C., Wolz, C., Harris, L., Berger-Bachi, B., and Bischoff, M. (2006) Staphylococcus aureus CcpA affects virulence determinant production and antibiotic resistance. Antimicrob Agents Chemother 50: 1183-1194.

Sharma, P., Haycocks, J.R.J., Middlemiss, A.D., Kettles, R.A., Sellars, L.E., Ricci, V., Piddock, L.J.V., and Grainger, D.C. (2017) The multiple antibiotic resistance operon of enteric bacteria controls DNA repair and outer membrane integ rity. Nature communications 8: 1444.

Shelburne, S.A., 3rd, Keith, D., Horstmann, N., Sumby, P., Davenport, M.T., Graviss, E.A., Brennan, R.G., and Musser, J.M. (2008) A direct link between carbohydrate utilization and virulence in the major human pathogen group A Streptococcus. Proc NatI Acad Sci USA 105: 1698-1703.

Shelburne, S.A., 3rd, Sahasrobhajane, P., Suber, B., Keith, D.B., Davenport, M.T., Horstmann, N., Kumaraswami, M., Olsen, R.J., Brennan, R.G., and Musser, J.M. (2011) Niche-specific contribution to streptococcal virulence of a MalR-regulated carbohydrate binding protein. Mol Microbio/ 81: 500-514.

Shelburne, S.A., Olsen, R.J., Suber, B., Sahasrabhojane, P., Sumby, P., Brennan, R.G., and Musser, J.M. (2010) A combination of independent transcriptional regulators shapes bacterial virulence gene expression during infection. PLoS Pathog 6: e1000817.

Sprehe, M., Seidel, G., Diel, M., and Hillen, W. (2007) CcpA mutants with differential activities in Bacillus subtilis. J Mol Microbiol Biotechnol 12: 96-105.

Stulke, J., and Hillen, W. (2000) Regulation of carbon catabolism in Bacillus species. Annu Rev Microbiol 54: 849-880.

Sumby, P., Porcella, S.F., Madrigal, A.G., Barbian, K.D., Virtaneva, K., Ricklefs, S.M., Sturdevant, D.E., Graham, M.R., Vuopio-Varkila, J., Hoe, N.P., and Musser, J.M. (2005) Evolutionary origin and emergence of a highly successful clone of serotype M1 group A Streptococcus involved multiple horizontal gene transfer events. J Infect Dis 192: 771-782.

Sumby, P., Whitney, A.R., Graviss, E.A., DeLeo, F.R., and Musser, J.M. (2006) Genome-wide analysis of group $A$ streptococci reveals a mutation that modulates global phenotype and disease specificity. PLoS Pathog 2: e5.

Sundar, G.S., Islam, E., Gera, K., Le Breton, Y., and Mclver, K.S. (2017) A PTS Ell mutant library in Group A Streptococcus identifies a promiscuous man-family PTS transporter influencing SLS-mediated hemolysis. Mol Microbiol 103: 518-533.

Thorvaldsdottir, H., Robinson, J.T., and Mesirov, J.P. (2013) Integrative Genomics Viewer (IGV): highperformance genomics data visualization and exploration. Brief Bioinform 14: 178-192.

Titgemeyer, F., and Hillen, W. (2002) Global control of sugar metabolism: a gram-positive solution. Antonie Van Leeuwenhoek 82: 59-71.

Vadeboncoeur, C., and Pelletier, M. (1997) The phosphoenolpyruvate:sugar phosphotransferase system of oral streptococci and its role in the control of sugar metabolism. FEMS Microbiol Rev 19: 187207.

Varga, J., Stirewalt, V.L., and Melville, S.B. (2004) The CcpA protein is necessary for efficient sporulation and enterotoxin gene (cpe) regulation in Clostridium perfringens. J Bacteriol 186: 5221-5229. 
Vega, L.A., Malke, H., and Mclver, K.S., (2016) Virulence-Related Transcriptional Regulators of Streptococcus pyogenes. In: Streptococcus pyogenes : Basic Biology to Clinical Manifestations. J.J. Ferretti, D.L. Stevens \& V.A. Fischetti (eds). Oklahoma City (OK), pp.

Walker, M.J., Barnett, T.C., McArthur, J.D., Cole, J.N., Gillen, C.M., Henningham, A., Sriprakash, K.S., Sanderson-Smith, M.L., and Nizet, V. (2014) Disease manifestations and pathogenic mechanisms of Group A Streptococcus. Clin Microbiol Rev 27: 264-301.

Warner, J.B., and Lolkema, J.S. (2003) CcpA-dependent carbon catabolite repression in bacteria. Microbiol Mol Biol Rev67: 475-490.

Watson, M.E., Jr., Nielsen, H.V., Hultgren, S.J., and Caparon, M.G. (2013) Murine vaginal colonization model for investigating asymptomatic mucosal carriage of Streptococcus pyogenes. Infect Immun 81: 1606-1617.

Willenborg, J., de Greeff, A., Jarek, M., Valentin-Weigand, P., and Goethe, R. (2014) The CcpA regulon of Streptococcus suis reveals novel insights into the regulation of the streptococcal central carbon metabolism by binding of CcpA to two distinct binding motifs. Mol Microbiol 92: 61-83.

Yang, Y., Zhang, L., Huang, H., Yang, C., Yang, S., Gu, Y., and Jiang, W. (2017) A Flexible Binding Site Architecture Provides New Insights into CcpA Global Regulation in Gram-Positive Bacteria. mBio 8.

Zeng, L., Choi, S.C., Danko, C.G., Siepel, A., Stanhope, M.J., and Burne, R.A. (2013) Gene regulation by CcpA and catabolite repression explored by RNA-Seq in Streptococcus mutans. PLoS One 8: e60465. 
bioRxiv preprint doi: https://doi.org/10.1101/2020.08.28.272682; this version posted August 28, 2020. The copyright holder for this preprint (which was not certified by peer review) is the author/funder. All rights reserved. No reuse allowed without permission. 